\title{
Binary collisions of charged particles in a magnetic field
}

\author{
H. B. Nersisyan* and G. Zwicknage $\dagger$ \\ Institut für Theoretische Physik II, Erlangen-Nürnberg Universität, Staudtstr. 7, D-91058 Erlangen, Germany
}

(Dated: June 6, 2022)

\begin{abstract}
Binary collisions between charged particles in an external magnetic field are considered in secondorder perturbation theory, starting from the unperturbed helical motion of the particles. The calculations are done with the help of an improved binary collisions treatment which is valid for any strength of the magnetic field, where the second-order energy and velocity transfers are represented in Fourier space for arbitrary interaction potentials. The energy transfer is explicitly calculated for a regularized and screened potential which is both of finite range and non-singular at the origin, and which involves as limiting cases the Debye (i.e., screened) and Coulomb potential. Two distinct cases are considered in detail. (i) The collision of two identical (e.g., electron-electron) particles; (ii) and the collision between a magnetized electron and an uniformly moving heavy ion. The energy transfer involves all harmonics of the electron cyclotron motion. The validity of the perturbation treatment is evaluated by comparing with classical trajectory Monte-Carlo calculations which also allows to investigate the strong collisions with large energy and velocity transfer at low velocities. For large initial velocities on the other hand, only small velocity transfers occur. There the non-perturbative numerical classical trajectory Monte-Carlo results agree excellently with the predictions of the perturbative treatment.
\end{abstract}

PACS numbers: $03.65 . \mathrm{Nk}, 34.50 . \mathrm{Bw}, 52.20 . \mathrm{Hv}, 52.40 . \mathrm{Mj}$

\section{INTRODUCTION}

In the presence of an external magnetic field $\mathbf{B}$ the problem of two charged particles cannot be solved in a closed form as the relative motion and the motion of the center of mass are coupled to each other. Therefore no theory exists for a solution of this problem that is uniformly valid for any strength of the magnetic field and the Coulomb force between the particles. The energy loss of ion beams and the related processes in a magnetized plasmas which are important in many areas of physics such as transport, heating, magnetic confinement of thermonuclear plasmas and astrophysics are examples of physical situations where this problem arises. Recent applications are the cooling of heavy ion beams by electrons $[1,[2,[3,4,5]$ and the energy transfer for heavy-ion inertial confinement fusion (ICF) (see, e.g., [6] for an overview). The classical limit of a hydrogen or Rydberg atom in a strong magnetic field also falls in this category (see, e.g., [7] and references therein) but in contrast to the free-free transitions (scattering) the total energy is negative here.

Numerical calculations have been performed for binary collisions (BC) between magnetized electrons $[\underline{8}, 9]$ and for collisions between magnetized electrons and ions 10, 11, 12, 13, 14]. In general the total energy $E$ of the particles interacting in a magnetic field is conserved but the relative and center of mass energies are not conserved separately. In addition, the presence of the magnetic field breaks the rotational symmetry of the system and as a consequence only the component of the angular momentum $\mathbf{L}$ parallel to the magnetic field $L_{\|}$is a constant of motion. So, the constants of motion $E$ and $L_{\|}$reduce the phase space of the relative motion. A different situation arises for the BC between an electron and uniformly moving heavy ion. As an ion is much heavier than an electron, its uniform motion is only weakly perturbed by collisions with the electrons and the magnetic field. In this case $L_{\|}$is not conserved but there exists a conserved generalized energy $K$ [12, 14] involving the energy of relative motion and a magnetic term. The apparently simple problem of charged particle interaction in a magnetic field is in fact a problem of considerable complexity and the additional degree of freedom of the cyclotron orbital motion produces a chaotic system with two degrees (or one degree for heavy ions) of freedom [14, 15, 16, 17].

In this paper we consider the BC between two charged particles treating the interaction (Coulomb) as a perturbation to their helical motions. For electron-heavy ion collisions this has been done previously in first order in the ion charge $Z$ and for an ion at rest [18] and in up to $\mathrm{O}\left(Z^{2}\right)$ for uniformly moving heavy ion [19, 20]. In Ref. [19] three regimes are identified, depending on the relative size of the parameters $a$ (the cyclotron radius), $s$ (the distance of the closest approach), and $\delta$ (the pitch of the helix). In earlier kinetic approaches [1, 2, 3, 4, 5] only two regimes have been

\footnotetext{
*Permanent address: Institute of Radiophysics and Electronics, 378410 Ashtarak, Armenia; Electronic address: hrachya@irphe.am ${ }^{\dagger}$ Electronic address: guenter.zwicknagel@physik.uni-erlangen.de
} 
distinguished: Fast collisions for $s<a$, where the Coulomb interaction is dominant and adiabatic collisions for $s>a$, where the magnetic field is important, as the electron performs many gyrations during the collision with the ion. The change $\Delta E_{i}$ of the energy of the ion has been related to the square of the momentum transfer $\Delta p$, which has been calculated up to $\mathrm{O}(Z)$. This is somewhat unsatisfactory, as there is another $\mathrm{O}\left(Z^{2}\right)$ contribution to $\Delta E_{i}$, in which the second-order momentum transfer enters linearly. Moreover, for applications in plasma physics (e.g., for calculation of the ion energy loss in a magnetized plasma) one calculates the angular averaged energy transfer which vanishes within first-order perturbation theory due to symmetry reasons and the ion energy change receives contribution only from higher orders [20]. Indeed, the transport phenomena, etc., are of order $\mathrm{O}\left(Z^{2}\right)$ in the ion charge.

Here we focus on BC between two magnetized identical particles (e.g. electrons) within the second order perturbation theory and its comparison with classical trajectory Monte-Carlo (CTMC) simulations. The present work considerably extends our earlier studies in Refs. [14, 20] where the second-order energy transfer for an ion-electron collision was calculated with the help of an improved $\mathrm{BC}$ treatment which is valid for any strength of the magnetic field and does not require the specification of the interaction potential. In addition, we consider here the impact parameter-averaged energy transfer for the $\mathrm{BC}$ between magnetized electron and heavy ion moving uniformly along the magnetic field which has not been yet considered in Refs. 14, 20], and will give new analytical expressions which are more appropriate for an explicit calculation of the energy loss. Physically these two distinct cases are similar except the time-dependent center of mass cyclotron motion in the case of two identical particles. The paper is organized as follows. In Sec. \starting from the exact equation of motion of two charged particles moving in a magnetic field we discuss some basic results of the exact BC treatment for the energy and velocity transfers as well as the energy conservation. In the following Sec. III, we discuss the velocity and energy transfer during BC of magnetized particles for arbitrary magnetic fields and strengths of the two-particle interaction potential. The equations of motion are solved in a perturbative manner up to the second order in interaction force starting from the unperturbed helical motion of the particles in a magnetic field. Then in Sec. IV we turn to the explicit calculation of the second order energy transfer for electron-electron collision but without any restriction on the magnetic field $\mathbf{B}$. The obtained energy transfer involves all cyclotron harmonics of the electron helical motion. For further applications (e.g., in cooling of ion beams, transport phenomena in magnetized plasmas) we consider the regularized and screened interaction potential which is both of finite range and less singular than the Coulomb interaction at the origin and as the limiting cases involves the Debye (i.e., screened) and Coulomb potentials. An exact solution for two particle collision in the presence of infinitely strong magnetic field is considered in Sec. V. This also suggests an improved perturbative treatment for repulsive interaction and in the case of strong magnetic field. In Sec. VI the results of the perturbative binary collision model are compared with CTMC simulations in which the scattering of ensembles of magnetized electrons are treated exactly. This comparison allows to determine clearly the range of validity of the perturbative treatment. The results are summarized and discussed in Sec. VII. Some details of the calculation of the generalized Coulomb logarithm is described in the Appendix $\mathrm{A}$. The small velocity limits of the energy transfers are derived in the Appendix B.

\section{BINARY COLLISION FORMULATION. GENERAL TREATMENT}

For our description of binary collision (BC) we start with considering the equations of motion for two charged particles moving in a homogeneous magnetic field and the related conservation laws, in general. This will then be specified to the two particular case on with we will focus in this paper. The BC between two electrons and between an electron and an uniformly moving heavy ion. Next the quantities of interest, the velocity transfer and the energy transfer of particles during the binary collision, will be introduced and discussed, before we turn to the solution of the equations of motion in the subsequent section.

\section{A. Relative and $\mathrm{cm}$ motion and conservation laws}

We consider two point charges with masses $m_{1}, m_{2}$ and charges $q_{1} e, q_{2} e$, respectively, moving in a homogeneous magnetic field $\mathbf{B}=B \mathbf{b}$ and interacting with the potential $q_{1} q_{2} \phi^{2} U(\mathbf{r})$ with $\phi^{2}=e^{2} / 4 \pi \varepsilon_{0}$. Here $\varepsilon_{0}$ is the permittivity of the vacuum and $\mathbf{r}=\mathbf{r}_{1}-\mathbf{r}_{2}$ is the relative coordinate of the colliding particles. For two isolated charged particles this interaction is given by the Coulomb potential, i.e. $U_{\mathrm{C}}(\mathbf{r})=1 / r$. In plasma applications the infinite range of this potential is modified by the screening. Then $U$ may be modeled by $U_{\mathrm{D}}(\mathbf{r})=e^{-r / \lambda} / r$ with a screening length $\lambda$ which can be chosen as the Debye screening length $\lambda_{\mathrm{D}}$, see, for example [21]. The quantum uncertainty principle prevents particles (for $q_{1} q_{2}<0$ ) from falling into the center of these potentials. In a classical picture this can be achieved by regularization of $U(\mathbf{r})$ at the origin, taking for example $U_{\mathrm{R}}(\mathbf{r})=\left(1-e^{-r / \lambda}\right) e^{-r / \lambda} / r,[22,23]$. Here $\lambda$ is a parameter, which is usually related to the de Broglie wavelength. 
In the presence of an external magnetic field, the Lagrangian and the corresponding equations of motion cannot be separated into parts describing the relative motion $\left[\mathbf{r}=\mathbf{r}_{1}-\mathbf{r}_{2}, \mathbf{v}=\dot{\mathbf{r}}\right]$ and the motion of the center of mass $(\mathrm{cm})\left[\mathbf{R}=\left(m_{1} \mathbf{r}_{1}+m_{2} \mathbf{r}_{2}\right) /\left(m_{1}+m_{2}\right), \mathbf{V}=\dot{\mathbf{R}}\right]$, in general (see, e.g., [8, 12, 19, 20]). Introducing the reduced mass $1 / \mu=1 / m_{1}+1 / m_{2}$ the equations of motion are

$$
\begin{aligned}
\dot{\mathbf{v}}(t)-\mu e B\left(\frac{q_{1}}{m_{1}^{2}}+\frac{q_{2}}{m_{2}^{2}}\right)[\mathbf{v}(t) \times \mathbf{b}] & =e B\left(\frac{q_{1}}{m_{1}}-\frac{q_{2}}{m_{2}}\right)[\mathbf{V}(t) \times \mathbf{b}]+\frac{q_{1} q_{2} \phi^{2}}{\mu} \mathbf{F}(\mathbf{r}(t)), \\
\dot{\mathbf{V}}(t)-e B\left(\frac{q_{1}+q_{2}}{m_{1}+m_{2}}\right)[\mathbf{V}(t) \times \mathbf{b}] & =\frac{\mu e B}{m_{1}+m_{2}}\left(\frac{q_{1}}{m_{1}}-\frac{q_{2}}{m_{2}}\right)[\mathbf{v}(t) \times \mathbf{b}],
\end{aligned}
$$

where $q_{1} q_{2} \phi^{2} \mathbf{F}(\mathbf{r}(t))(\mathbf{F}=-\partial U / \partial \mathbf{r})$ is the force exerted by the particle 2 on the particle 1 . The coupled, nonlinear differential equations (11) and (2) completely describe the motion of the particles. For solving the scattering problem, they have to be integrated numerically for a complete set of the initial conditions.

From Eqs. (1) and (2) follows the conservation of the parallel component of the cm velocity $\mathbf{V}(t) \cdot \mathbf{b}=V_{0 \|}$ and the total energy

$$
E=E_{\mathrm{cm}}+E_{\mathrm{r}}=\frac{\left(m_{1}+m_{2}\right) V^{2}(t)}{2}+\frac{\mu v^{2}(t)}{2}+q_{1} q_{2} \phi^{2} U(\mathbf{r})=\mathrm{const},
$$

but since, in general, the relative and center of mass motions are coupled the relative $E_{\mathrm{r}}$ and $\mathrm{cm} E_{\mathrm{cm}}$ energies are not conserved separately.

In the case of two identical particles, taking here electrons, i.e. $m_{1}=m_{2}=m, q_{1}=q_{2}=-1$, the equations of motion considerably simplify to

$$
\begin{aligned}
\dot{\mathbf{v}}(t)+\omega_{c}[\mathbf{v}(t) \times \mathbf{b}] & =\frac{2 \phi^{2}}{m} \mathbf{F}(\mathbf{r}(t)), \\
\dot{\mathbf{V}}(t)+\omega_{c}[\mathbf{V}(t) \times \mathbf{b}] & =0
\end{aligned}
$$

with the cyclotron frequency of the electron $\omega_{c}=e B / m$. Here the cm-motion, Eq. (5), can be solved which leads to

$$
\mathbf{V}(t) \equiv \mathbf{V}_{0}(t)=V_{0 \|} \mathbf{b}+V_{0 \perp}\left[\mathbf{u}_{c} \cos \left(\omega_{c} t\right)+\left[\mathbf{b} \times \mathbf{u}_{c}\right] \sin \left(\omega_{c} t\right)\right]
$$

where $V_{0 \perp} \geqslant 0$ is the cm velocity transverse to the magnetic field direction $\mathbf{b}$, and $\mathbf{u}_{c}=\left(\cos \varphi_{c}, \sin \varphi_{c}\right)$ is the unit vector perpendicular to $\mathbf{b}\left(\varphi_{c}\right.$ is the phase of the $\mathrm{cm}$ transversal motion which is fixed by initial conditions). The velocities $V_{0 \|}$ and $V_{0 \perp}$ are related to the particles unperturbed parallel $v_{0 \nu \|}$ and transverse $v_{0 \nu \perp}$ velocities $(\nu=1,2)$

$$
V_{0 \|}=\frac{v_{01 \|}+v_{02 \|}}{2}, \quad V_{0 \perp} \mathbf{u}_{c}=\frac{v_{01 \perp} \mathbf{u}_{1}+v_{02 \perp} \mathbf{u}_{2}}{2}
$$

with the unit vectors $\mathbf{u}_{\nu}=\left(\cos \varphi_{\nu}, \sin \varphi_{\nu}\right)\left(\varphi_{\nu}\right.$ are the initial phases of the particles $)$ which fix the initial transverse velocities (or coordinates) of the particles 1 and 2 .

With the help of the equation of motion (4) and relation (6) it can be easily proven that the relative $\left(E_{\mathrm{r}}\right)$ and $\mathrm{cm}$ $\left(E_{\mathrm{cm}}\right)$ energies

$$
E_{\mathrm{cm}}=m \mathbf{V}_{0}^{2}(t)=m\left(V_{0 \|}^{2}+V_{0 \perp}^{2}\right), \quad E_{\mathrm{r}}=\frac{m v^{2}}{4}+\not^{2} U(\mathbf{r})
$$

are here conserved separately. They can be expressed by the particles initial (unperturbed) velocities $\mathbf{v}_{0 \nu}$ according to $E_{\mathrm{cm}}=\frac{m}{4}\left(\mathbf{v}_{01}+\mathbf{v}_{02}\right)^{2}, E_{\mathrm{r}}=\frac{m}{4}\left(\mathbf{v}_{01}-\mathbf{v}_{02}\right)^{2}$. Note that $E_{\mathrm{cm}}$ and $E_{\mathrm{r}}$ are here functions only of the difference of the initial phases of the particles $\varphi=\varphi_{1}-\varphi_{2}$.

In the second case of BC between an electron and an heavy ion of mass $M$ and charge $Z e$, i.e. $q_{1}=-1, q_{2}=Z$, $m_{1}=m, m_{2}=M$, and assuming $m / M \rightarrow 0, \mu \rightarrow m$, the equations of motion (11) and (2) turn into

$$
\begin{aligned}
\dot{\mathbf{v}}(t)+\omega_{c}[\mathbf{v}(t) \times \mathbf{b}] & =-\omega_{c}\left[\mathbf{v}_{i} \times \mathbf{b}\right]-\frac{Z \phi^{2}}{m} \mathbf{F}(\mathbf{r}(t)), \\
\dot{\mathbf{V}}(t) & =0,
\end{aligned}
$$

where $\mathbf{v}_{i}$ is the given heavy ion velocity and $\mathbf{V}(t)=\mathbf{v}_{i}=$ const. While the relative energy $E_{\mathrm{r}}$ itself is not conserved in this case, there exists nevertheless a constant of motion

$$
K=\frac{m v^{2}}{2}-Z \phi^{2} U(\mathbf{r})+m \omega_{c} \mathbf{r} \cdot\left[\mathbf{v}_{i} \times \mathbf{b}\right]=E_{\mathrm{r}}+m \omega_{c} \mathbf{r} \cdot\left[\mathbf{v}_{i} \times \mathbf{b}\right]
$$


which can be easily checked with the help of Eq. (9). In contrast to the unmagnetized case, the relative energy transfer during an ion-electron collision is thus proportional to $\delta r_{\perp} v_{i \perp}$, where $\delta r_{\perp}$ and $v_{i \perp}$ are the perpendicular components of the change of relative position and the ion velocity. Only for ions which move along the magnetic field direction, i.e. $\left(\mathbf{v}_{i \perp}=0\right)$, where the magnetic term in Eq. (11) vanishes, the relative energy is conserved. This case is very similar to the case of electron-electron collisions except that the cm velocity is constant.

\section{B. Energy loss and velocity transfer}

In the general case the rate $d E_{\nu} / d t$ at which the energy $E_{\nu}=m_{\nu} v_{\nu}^{2} / 2$ of particle $\nu$ changes during the collision with the other particle can be obtained by multiplying the equation of motion for particle $\nu$ by its velocity $\mathbf{v}_{\nu}(t)=$ $\mathbf{V}(t)+\varrho_{\nu}\left(\mu / m_{\nu}\right) \mathbf{v}(t)$, where $\varrho_{1}=1, \varrho_{2}=-1$. As $q_{1} q_{2} \phi^{2} \mathbf{F}(\mathbf{r})$ is the force exerted by the particle 2 on the particle 1 the integration of this rate over the whole collision yields the energy transfer (see, 14] for further details)

$$
\Delta E_{\nu}=\varrho_{\nu} q_{1} q_{2} \phi^{2} \int_{-\infty}^{\infty} \mathbf{V}(\tau) \cdot \mathbf{F}(\mathbf{r}(\tau)) d \tau
$$

assuming that for $t \rightarrow \pm \infty, r(t) \rightarrow \infty$ and $U(\mathbf{r}(t)) \rightarrow 0$. According to the conservation of total energy we have $\Delta E_{1}=-\Delta E_{2}$, as it can be directly seen from Eq. (12).

Alternatively the energy changes (12) can be expressed by the velocity transferred to particle 1 (or 2) during the collision. For this purpose we split the velocity of the $\nu$ th particle, $\mathbf{v}_{\nu}(t)$, into $\mathbf{v}_{\nu}(t)=\mathbf{v}_{0 \nu}(t)+\delta \mathbf{v}_{\nu}(t)$. Here $\mathbf{v}_{0 \nu}(t)$ describes the free helical motion of the particles in the magnetic field, $\dot{\mathbf{v}}_{0 \nu}-\left(q_{\nu} e B / m_{\nu}\right)\left[\mathbf{v}_{0 \nu} \times \mathbf{b}\right]=0$, while $\delta \mathbf{v}_{\nu}(t)$ is the related velocity change (with $\delta \mathbf{v}_{\nu}(t \rightarrow-\infty) \rightarrow 0$ ). From the energy change with respect to the free motion $\delta E_{\nu}(t)=\left(m_{\nu} / 2\right)\left\{\left[\mathbf{v}_{0 \nu}(t)+\delta \mathbf{v}_{\nu}(t)\right]^{2}-\mathbf{v}_{0 \nu}^{2}(t)\right\}$ the total energy transfer $\Delta E_{\nu}$ can then be expressed through the total velocity transfer $\Delta \mathbf{v}_{\nu}=\delta \mathbf{v}_{\nu}(t \rightarrow \infty)=\left[\mathbf{v}_{\nu}(t)-\mathbf{v}_{0 \nu}(t)\right]_{t \rightarrow \infty}$ and $\mathbf{v}_{0 \nu} \cdot \Delta \mathbf{v}_{\nu}=\left.\mathbf{v}_{0 \nu}(t) \cdot \delta \mathbf{v}_{\nu}(t)\right|_{t \rightarrow \infty}$

$$
\Delta E_{1}=-\Delta E_{2}=m_{1}\left(\mathbf{v}_{01} \cdot \Delta \mathbf{v}_{1}+\frac{1}{2} \Delta \mathbf{v}_{1}^{2}\right) .
$$

Employing this way of calculating the energy transfer, as e.g. in [19], the potential $U(\mathbf{r})$ has to be specified already at an early stage of the calculation. In Sec. [II we will show that Eq. (12) allows for a more general formulation in which the cut-off at large distances and the regularization at small distances can be treated much easier.

So far we considered the energy transfers of the particles in the laboratory frame. In addition the energy transfers $\Delta E_{1}$ and $\Delta E_{2}$ can be expressed by the change of the relative energy $\Delta E_{\mathrm{r}}=\Delta\left(\mu \mathbf{v}^{2} / 2\right)$ and the relative and $\mathrm{cm}$ velocity transfer $\Delta[\mathbf{V} \cdot \mathbf{v}]$, respectively. Since $\Delta E_{\mathrm{r}}=-\Delta E_{\mathrm{cm}}$ (due to the conservation of total energy), there follows a relation between the energy transfers $\Delta E_{1}$ and $\Delta E_{\mathrm{r}}$ given by

$$
\Delta E_{1}=\frac{m_{2}-m_{1}}{m_{2}+m_{1}} \Delta E_{\mathrm{r}}+\mu \Delta[\mathbf{V} \cdot \mathbf{v}] .
$$

Here, the second term is the total change of the scalar product $\mathbf{V}(t) \cdot \mathbf{v}(t)$. For instance, this quantity is a constant for the free, unperturbed motion of identical particles where $\mathbf{V}_{0}(t) \cdot \mathbf{v}_{0}(t)=\left(v_{01}^{2}-v_{02}^{2}\right) / 2=$ const and is given by the initial velocities $\mathbf{v}_{01}, \mathbf{v}_{02}$.

In the case of identical particles the relative energy and the cm energy are conserved, i.e. $\Delta E_{r}=-\Delta E_{\mathrm{cm}}=0$, and thus $\Delta E_{1}=-\Delta E_{2}=\mu \Delta[\mathbf{V} \cdot \mathbf{v}]$. We note however that the longitudinal $\left(\Delta E_{r \|}\right)$ and transverse $\left(\Delta E_{r \perp}=-\Delta E_{r \|}\right)$ parts of the energy transfer $\Delta E_{r}$ do not vanish, where

$$
\Delta E_{r \|}=\frac{m}{4} \Delta v_{\|}\left(2 v_{r \|}+\Delta v_{\|}\right) \neq 0 .
$$

Here $v_{r \|}=v_{01 \|}-v_{02 \|}$ and $\Delta v_{\|}$are the components of $\mathbf{v}_{0}(t)$ and $\Delta \mathbf{v}$ parallel to $\mathbf{b}$, respectively.

For the collision of an electron and a heavy ion, with $m_{2}=M \gg m=m_{1}$ and $\mathbf{V}=\mathbf{v}_{i}$, the energy transfer to the ion $\Delta E_{i}=\Delta E_{2}=-\Delta E_{1}$ then follows from Eq. (14) as

$$
\Delta E_{i}=-\Delta E_{r}-m \mathbf{v}_{i} \cdot \Delta \mathbf{v}=-\frac{m}{2} \Delta\left(\mathbf{v}^{2}\right)-m \mathbf{v}_{i} \cdot \Delta \mathbf{v} .
$$

When the ion moves parallel to the magnetic field direction the relative energy transfer in Eq. (16) vanishes and $\Delta E_{i \|}=-m v_{i \|} \Delta v_{\|}$, where $v_{i \|}$ is the component of the ion velocity parallel to $\mathbf{b}$. 


\section{PERTURBATIVE TREATMENT. GENERAL THEORY}

\section{A. Trajectory correction}

In this section we consider the theoretical treatment of the scattering of two identical particle, here electrons, where we seek an approximate solution of Eq. (4) by assuming the interaction force between the particles as a perturbation to the free helical motion. For the case of ion-electron scattering the corresponding considerations and derivations are discussed in detail in Ref. [14] and we thus focus on the electron-electron case in the forthcoming discussion.

As the velocity of the $\mathrm{cm}$ motion is already fixed by Eq. (6), we have to look for the solution of Eq. (4) for the variables $\mathbf{r}$ and $\mathbf{v}$ in a perturbative manner

$$
\mathbf{r}(t)=\mathbf{r}_{0}(t)+\mathbf{r}^{(1)}(t)+\mathbf{r}^{(2)}(t) \ldots, \quad \mathbf{v}(t)=\mathbf{v}_{0}(t)+\mathbf{v}^{(1)}(t)+\mathbf{v}^{(2)}(t) \ldots
$$

where $\mathbf{r}_{0}(t), \mathbf{v}_{0}(t)$ are the unperturbed two-particles relative coordinate and velocity, respectively, $\mathbf{r}^{(n)}(t), \mathbf{v}^{(n)}(t) \propto$ $q^{2 n} \mathbf{F}_{n-1}(n=1,2, \ldots)$ are the $n$th order perturbations of $\mathbf{r}(t)$ and $\mathbf{v}(t)$, which are proportional to $q^{2 n}$ (for electrons $q=-1) . \mathbf{F}_{n}(t)$ is the $n$th order correction to the two-particle interaction force. Using the expansion (17) for the $n$th order corrections $\mathbf{F}_{n}$ we obtain

$$
\mathbf{F}(\mathbf{r}(t))=\mathbf{F}_{0}\left(\mathbf{r}_{0}(t)\right)+\mathbf{F}_{1}\left(\mathbf{r}_{0}(t), \mathbf{r}_{1}(t)\right)+\ldots
$$

where

$$
\begin{gathered}
\mathbf{F}_{0}\left(\mathbf{r}_{0}(t)\right)=\mathbf{F}\left(\mathbf{r}_{0}(t)\right)=-i \int d \mathbf{k} U(\mathbf{k}) \mathbf{k} e^{i \mathbf{k} \cdot \mathbf{r}_{0}(t)}, \\
\mathbf{F}_{1}\left(\mathbf{r}_{0}(t), \mathbf{r}_{1}(t)\right)=\left.\left(\mathbf{r}_{1}(t) \cdot \frac{\partial}{\partial \mathbf{r}}\right) \mathbf{F}(\mathbf{r})\right|_{\mathbf{r}=\mathbf{r}_{0}(t)}=\int d \mathbf{k} U(\mathbf{k}) \mathbf{k}\left[\mathbf{k} \cdot \mathbf{r}_{1}(t)\right] e^{i \mathbf{k} \cdot \mathbf{r}_{0}(t)} .
\end{gathered}
$$

In Eqs. (19) and (20), we have introduced the two-particle interaction potential $U(\mathbf{r})$ through $\mathbf{F}(\mathbf{r})=-\partial U(\mathbf{r}) / \partial \mathbf{r}$ and the force corrections have been written using a Fourier transformation in space.

We start with the zero-order unperturbed helical motion of two electrons in the relative frame

$$
\begin{aligned}
& \mathbf{r}_{0}(t)=\mathbf{R}_{0}+\mathbf{b} v_{r \|} t+a\left[\mathbf{u}_{r} \sin \left(\omega_{c} t\right)-\left[\mathbf{b} \times \mathbf{u}_{r}\right] \cos \left(\omega_{c} t\right)\right], \\
& \mathbf{v}_{0}(t)=\dot{\mathbf{r}}_{0}(t),
\end{aligned}
$$

where $\mathbf{u}_{r}=\left(\cos \varphi_{r}, \sin \varphi_{r}\right)\left(\varphi_{r}\right.$ is the initial phase of the relative motion) is the unit vector perpendicular to the magnetic field, $v_{r \|}=v_{01 \|}-v_{02 \|}$ and $v_{0 \perp} \mathbf{u}_{r}=v_{01 \perp} \mathbf{u}_{1}-v_{02 \perp} \mathbf{u}_{2}$ (with $v_{0 \perp} \geqslant 0$ ) are the unperturbed relative velocity components parallel and perpendicular to $\mathbf{b}$, respectively. Here $v_{r \|} \mathbf{b}$ is the relative velocity of the guiding centers of two particles, and $a=v_{0 \perp} / \omega_{c}$ is the relative cyclotron radius. It should be noted that in Eq. (21) the variables $\mathbf{u}_{r}$ and $\mathbf{R}_{0}$ are independent and are defined by the initial conditions. Explicitly the relative cyclotron radius $a$ and the phase $\varphi_{r}$ are connected to the particles cyclotron radii $a_{1}, a_{2}$ and phases $\varphi_{1}, \varphi_{2}$, and $\varphi=\varphi_{1}-\varphi_{2}$, according to

$$
e^{i \varphi_{r}}=\frac{a_{1} e^{i \varphi_{1}}-a_{2} e^{i \varphi_{2}}}{a}, \quad a^{2}=a_{1}^{2}+a_{2}^{2}-2 a_{1} a_{2} \cos \varphi .
$$

The equation for the first-order relative velocity correction is given by

$$
\dot{\mathbf{v}}^{(1)}(t)+\omega_{c}\left[\mathbf{v}^{(1)}(t) \times \mathbf{b}\right]=\frac{2 q^{2} \phi^{2}}{m} \mathbf{F}\left(\mathbf{r}_{0}(t)\right)
$$

with the solution

$$
\begin{aligned}
& \mathbf{r}^{(1)}(t)=\frac{2 q^{2} \phi^{2}}{m}\left\{\mathbf{b} P_{\|}(t)+\operatorname{Re}\left[\mathbf{P}_{\perp}(t)-\mathbf{b}\left(\mathbf{b} \cdot \mathbf{P}_{\perp}(t)\right)-i\left[\mathbf{b} \times \mathbf{P}_{\perp}(t)\right]\right]\right\} . \\
& \mathbf{v}^{(1)}(t)=\dot{\mathbf{r}}^{(1)}(t) .
\end{aligned}
$$

Here we have assumed that all corrections vanish at $t \rightarrow-\infty$ and have introduced the following abbreviations

$$
\begin{array}{r}
P_{\|}(t)=-i \int d \mathbf{k} U(\mathbf{k})(\mathbf{k} \cdot \mathbf{b}) \int_{-\infty}^{t} d \tau(t-\tau) e^{i \mathbf{k} \cdot \mathbf{r}_{0}(\tau)}, \\
\mathbf{P}_{\perp}(t)=-\frac{1}{\omega_{c}} \int d \mathbf{k} U(\mathbf{k}) \mathbf{k} \int_{-\infty}^{t} d \tau\left[e^{i \omega_{c}(t-\tau)}-1\right] e^{i \mathbf{k} \cdot \mathbf{r}_{0}(\tau)},
\end{array}
$$

wherein $\mathbf{r}_{0}(t)$ is given by Eq. (21) and the Fourier representation of the interaction force, Eq. (19), has been used. 


\section{B. First and second order energy transfers}

The total energy change $\Delta E_{1}$ of the first particle during collision with the particle 2 is given by Eq. (12). Insertion of Eq. (18) into the general expression (12) yields

$$
\Delta E_{1}=\Delta E_{1}^{(1)}+\Delta E_{1}^{(2)}+\ldots
$$

where

$$
\Delta E_{1}^{(1)}=q^{2} \phi^{2} \int_{-\infty}^{\infty} d t \mathbf{V}_{0}(t) \cdot \mathbf{F}\left(\mathbf{r}_{0}(t)\right), \quad \Delta E_{1}^{(2)}=q^{2} \phi^{2} \int_{-\infty}^{\infty} d t \mathbf{V}_{0}(t) \cdot \mathbf{F}_{1}\left(\mathbf{r}_{0}(t), \mathbf{r}_{1}(t)\right)
$$

are the first- and second order energy transfer, respectively.

We now introduce the variable $\mathbf{s}=\mathbf{R}_{0 \perp}$ which is the component of $\mathbf{R}_{0}$ perpendicular to the relative velocity vector $v_{r \|}$ b. From Eq. (21) we can see that $\mathbf{s}$ is the distance of closest approach for the guiding centers of the two particles' helical motion. For practical applications the energy change is now given by the average of $\Delta E_{1}$ with respect to the initial phases of the particles $\varphi_{1}, \varphi_{2}$ and the azimuthal angle $\vartheta_{\mathbf{s}}$ of $\mathbf{s}$. Such an averaged quantity $f$ will be abbreviated by $\langle f\rangle$ in the forthcoming considerations.

We start with calculating the first order longitudinal relative velocity transfer, $\Delta v_{\|}^{(1)}$, which also contributes to the second order relative energy transfer according to Eq. (15). This quantity can be easily extracted from the parallel component of the first-order relative velocity correction, $\mathbf{b} \cdot \mathbf{v}^{(1)}(t)$, in the limit $t \rightarrow \infty$, i.e. after completion of the interaction. Thus, using the Fourier series of the exponential function $e^{i z \sin \theta}$ [24], we obtain from Eqs. (24) and (25)

$$
\Delta v_{\|}^{(1)}=-\frac{4 \pi i q^{2} \phi^{2}}{m} \int d \mathbf{k} U(\mathbf{k}) k_{\|} e^{i \mathbf{k} \cdot \mathbf{R}_{0}} \sum_{n=-\infty}^{\infty} e^{i n\left(\varphi_{r}-\theta\right)} J_{n}\left(k_{\perp} a\right) \delta\left(\zeta_{n}(\mathbf{k})\right) .
$$

Here $J_{n}$ are the Bessel functions of the $n$th order, $\zeta_{n}(\mathbf{k})=n \omega_{c}+k_{\|} v_{r \|}, k_{\|}=\mathbf{k} \cdot \mathbf{b}$ and $k_{\perp}$ are the components of $\mathbf{k}$ parallel and transverse to $\mathbf{b}$, respectively, $\tan \theta=k_{y} / k_{x}$. Note that the relative cyclotron radius $a$ as well as the velocity of the cm motion $V_{0 \perp}$ depend on $\varphi=\varphi_{1}-\varphi_{2}$ (see Eq. (7) and (22)). Performing the averages with respect to the initial phases $\varphi_{1}, \varphi_{2}$ and the azimuthal angle $\vartheta_{\mathbf{s}}$ results in $\left\langle\Delta v_{\|}^{(1)}\right\rangle=0$ for spherically symmetric interaction potentials $(U(\mathbf{r})=U(r)$ and $U(\mathbf{k})=U(k))$ (see, e.g., Refs. [14, 20]) due to symmetry.

The first-order energy transfer is obtained by substituting Eqs. (6), (19) and (21) into the first one of Eq. (27). Similarly as the first-order relative velocity correction $\Delta v_{\|}^{(1)}(28)$ the first-order energy change also vanishes if averaged with respect to the initial phases of the particles $\varphi_{1}, \varphi_{2}$ and $\vartheta_{\mathbf{s}}$.

Thus the energy change receives a contribution only from higher orders, and we next evaluate the second order energy transfer $\Delta E_{1}^{(2)}$ by inserting Eqs. (6), (20), (21), (24) and (25) into the second equation of Eq. (27). This quantity is then averaged with respect to the initial phases of the particles $\varphi_{1}, \varphi_{2}$ and the azimuthal angle $\vartheta_{\mathbf{s}}$ of the impact parameter $\mathbf{s}$. The obtained angular integrals are easily evaluated using the Fourier series of the exponential function. After averaging the energy transfer $\Delta E_{1}^{(2)}$ with respect to $\varphi_{1}$ and $\varphi_{2}$ the remaining part will depend on $\delta\left(\left(\mathbf{k}+\mathbf{k}^{\prime}\right) \cdot \mathbf{b}\right)$, i.e. the component of $\mathbf{k}+\mathbf{k}^{\prime}$ along the magnetic field $\mathbf{b}$. Thus this $\delta$-function enforces $\mathbf{k}+\mathbf{k}^{\prime}$ to lie in the plane transverse to $\mathbf{b}$ so that $e^{i\left(\mathbf{k}+\mathbf{k}^{\prime}\right) \cdot \mathbf{R}_{0}} \delta\left(\left(\mathbf{k}+\mathbf{k}^{\prime}\right) \cdot \mathbf{b}\right)=e^{i \mathbf{Q} \cdot \mathbf{s}} \delta\left(\left(\mathbf{k}+\mathbf{k}^{\prime}\right) \cdot \mathbf{b}\right)$, where $\mathbf{Q}=\mathbf{k}_{\perp}+\mathbf{k}_{\perp}^{\prime}$. In addition, instead of phase $\varphi_{1}$ we introduce a new variable $\varphi$ according to $\varphi_{1}=\varphi+\varphi_{2}$. Then after performing $\varphi_{2}$-integration there remains average with respect to the phase difference $\varphi$. The result of the angular averaging finally reads

$$
\left\langle\Delta E_{1}^{(2)}\right\rangle=\int_{0}^{2 \pi} \frac{d \varphi}{2 \pi} \mathcal{E}(\varphi, s),
$$

where

$$
\begin{aligned}
\mathcal{E}(\varphi, s)= & -\frac{2 \pi i q^{4} \phi^{4}}{m\left|v_{r \|}\right|} \int d \mathbf{k} d \mathbf{k}^{\prime} U(\mathbf{k}) U\left(\mathbf{k}^{\prime}\right) J_{0}(Q s) \delta\left(k_{\|}^{\prime}+k_{\|}\right) \sum_{n=-\infty}^{\infty}(-1)^{n} e^{i n\left(\theta-\theta^{\prime}\right)} \\
& \times J_{n}\left(k_{\perp}^{\prime} a\right) J_{n}\left(k_{\perp} a\right) G_{n}\left(\mathbf{k}, \mathbf{k}^{\prime}\right)\left(V_{0 \|} k_{\|}-n \omega_{c} \frac{V_{0 \perp}}{v_{0 \perp}} \cos \vartheta\right), \\
G_{n}\left(\mathbf{k}, \mathbf{k}^{\prime}\right)=- & \frac{2 k_{\|} k_{\|}^{\prime}}{\left(\zeta_{n}\left(\mathbf{k}^{\prime}\right)-i 0\right)^{2}}-\frac{k_{\perp} k_{\perp}^{\prime} e^{i\left(\theta^{\prime}-\theta\right)}}{\left(\zeta_{n}\left(\mathbf{k}^{\prime}\right)-i 0\right)\left(\zeta_{n-1}\left(\mathbf{k}^{\prime}\right)-i 0\right)}-\frac{k_{\perp} k_{\perp}^{\prime} e^{-i\left(\theta^{\prime}-\theta\right)}}{\left(\zeta_{n}\left(\mathbf{k}^{\prime}\right)-i 0\right)\left(\zeta_{n+1}\left(\mathbf{k}^{\prime}\right)-i 0\right)} .
\end{aligned}
$$


The phase factor $\vartheta=\varphi_{r}-\varphi_{c}$ in Eq. (30) can be evaluated explicitly by using Eqs. (77) and (22). Introducing the phase difference of the particles $\varphi=\varphi_{1}-\varphi_{2}$ it reads

$$
e^{i\left(\varphi_{r}-\varphi_{c}\right)} \equiv e^{i \vartheta}=\frac{a_{1}^{2}-a_{2}^{2}+2 i a_{1} a_{2} \sin \varphi}{\sqrt{\left(a_{1}^{2}-a_{2}^{2}\right)^{2}+4 a_{1}^{2} a_{2}^{2} \sin ^{2} \varphi}} .
$$

The series representation (30) of the second-order energy transfer is valid for any strength of the magnetic field.

For most applications it is also useful to integrate the $\varphi_{1}, \varphi_{2}, \vartheta_{\mathbf{s}}$-averaged energy transfer, $\left\langle\Delta E_{1}^{(2)}\right\rangle$, with respect to the impact parameters $s$ in the full 2D space. We thus introduce a generalized cross-section [10, 11, 14, 20] through the relation

$$
\sigma=\int_{0}^{\infty}\left\langle\Delta E_{1}^{(2)}\right\rangle s d s=\int_{0}^{2 \pi} \frac{d \varphi}{2 \pi} \bar{\sigma}(\varphi)=\int_{0}^{2 \pi} \frac{d \varphi}{2 \pi} \int_{0}^{\infty} \mathcal{E}(\varphi, s) s d s .
$$

As $\bar{\sigma}(\varphi)$ results from the $s$-integration of the energy transfer $\mathcal{E}(\varphi, s)$ one obtains an expression for $\bar{\sigma}(\varphi)$ which represents an infinite sum over Bessel functions. Moreover, assuming regularized interaction and performing kintegration in $\bar{\sigma}(\varphi)$ yields an infinite sum over modified Bessel functions (see, e.g., an example for ion-electron collision in Ref. [14]). For arbitrary axially symmetric interaction potential similar expression is derived in Appendix B (see Eq. (B1) $)$. However, for practical applications it is much more convenient to use an equivalent integral representation of the effective cross-section which does not involve any special function. This expression can be derived from the Bessel-function representation of the cross-section $\bar{\sigma}(\varphi)$ using the integral representation of the Dirac $\delta$-function as well as the summation formula for $\sum_{n} e^{i n \varphi} J_{n}^{2}(a)[24]$. The energy transfer $\bar{\sigma}(\varphi)$ after lengthy but straightforward calculations then reads

$$
\bar{\sigma}(\varphi)=\bar{\sigma}_{\|}(\varphi)+\bar{\sigma}_{\perp}(\varphi)
$$

with

$$
\begin{aligned}
\bar{\sigma}_{\|}(\varphi)= & -\frac{2(2 \pi)^{2} q^{4} \phi^{4} V_{0 \|}}{m \omega_{c}^{2} v_{r \|}} \int_{0}^{\infty} t d t \int d \mathbf{k}|U(\mathbf{k})|^{2} \\
& \times\left(k_{\|}^{2}+k_{\perp}^{2} \frac{\sin t}{t}\right) k_{\|} \sin \left(k_{\|} \delta t\right) J_{0}\left(2 k_{\perp} a \sin \frac{t}{2}\right), \\
\bar{\sigma}_{\perp}(\varphi)=- & \frac{2(2 \pi)^{2} q^{4} \phi^{4} V_{0 \perp}}{m \omega_{c}^{2}\left|v_{r \|}\right|} \cos \vartheta \int_{0}^{\infty} t d t \int d \mathbf{k}|U(\mathbf{k})|^{2} \\
& \times\left(k_{\|}^{2}+k_{\perp}^{2} \frac{\sin t}{t}\right) k_{\perp} \cos \left(k_{\|} \delta t\right) \cos \left(\frac{t}{2}\right) J_{1}\left(2 k_{\perp} a \sin \frac{t}{2}\right),
\end{aligned}
$$

where $\delta=\left|v_{r}\right| \mid / \omega_{c}$ is the relative pitch of the particles helices, divided by $2 \pi$. In Eq. (34) the cross-section $\bar{\sigma}(\varphi)$ has been splitted into two parts which correspond to the cm motion along $\left(\bar{\sigma}_{\|}(\varphi)\right)$ and transverse $\left(\bar{\sigma}_{\perp}(\varphi)\right)$ to the magnetic field.

An expression similar to Eqs. (29) and (30) has been obtained in [14, 20] for electron-heavy ion (no cyclotron motion) collision where the direction $\mathbf{b}$ of the magnetic field and the direction $\mathbf{n}_{r}=\mathbf{v}_{r} / v_{r}$ of the relative velocity $\mathbf{v}_{r}=v_{e \|} \mathbf{b}-\mathbf{v}_{i}\left(v_{e \|}\right.$ is the component of electron velocity parallel to the magnetic field) of the electron guiding center is singled out in the argument of the $\delta$-function and the summand of the $n$-summation. This prevents a closed evaluation of the energy transfer for ion-electron collision for arbitrary direction of the ion motion with respect to the magnetic field. But if the ion moves along the magnetic field and the guiding center $v_{r \|} \mathbf{b}$ has no component in transverse direction, as it is always the case for a collision of two identical gyrating particles, the energy transfer in such ion-electron collisions can be evaluated in the same manner as already discussed in context with deriving Eqs. (34)-(36) in a straightforward manner.

For that case of ion-electron collisions with constant $\mathbf{v}_{i}=v_{i \|} \mathbf{b}$ the energy loss of the ion is given by $\Delta E_{i}=$ $-m v_{i \|} \Delta v_{\|}$, see Eq. (16), because the relative energy transfer $\Delta E_{r}$ here vanishes according to Eq. (11). The velocity transfer $\left\langle\Delta v_{\|}^{(2)}\right\rangle$ required for $\left\langle\Delta E_{i}^{(2)}\right\rangle$ can be extracted from Eqs. (29) and (30). For identical particles this is the term in Eq. (30) which is proportional to $V_{0 \|}$ (see, e.g., Eq. (14)), i.e.

$$
\left\langle\Delta v_{\|}^{(2)}\right\rangle=\int_{0}^{2 \pi} \frac{d \varphi}{2 \pi} \mathcal{U}_{1}(\varphi, s)
$$


with

$$
\begin{aligned}
\mathcal{U}_{1}(\varphi, s)= & -\frac{4 \pi i q^{4} \phi^{4}}{m^{2}\left|v_{r \|}\right|} \int d \mathbf{k} d \mathbf{k}^{\prime} U(\mathbf{k}) U\left(\mathbf{k}^{\prime}\right) k_{\|} J_{0}(Q s) \delta\left(k_{\|}^{\prime}+k_{\|}\right) \\
& \times \sum_{n=-\infty}^{\infty}(-1)^{n} e^{i n\left(\theta-\theta^{\prime}\right)} J_{n}\left(k_{\perp} a\right) J_{n}\left(k_{\perp}^{\prime} a\right) G_{n}\left(\mathbf{k}, \mathbf{k}^{\prime}\right) .
\end{aligned}
$$

For the present case of these specific ion-electron collisions the quantities $q^{4}$ and $\mu=m / 2$ in Eq. (38) have to be replaced with $Z^{2}$ and $m$, respectively. Thus the $s$-integrated $\left\langle\Delta v_{\|}^{(2)}\right\rangle$ for ion-electron collisions corresponds to Eq. (35), i.e. more precisely to

$$
\int_{0}^{\infty}\left\langle\Delta v_{\|}^{(2)}\right\rangle s d s=\frac{Z^{2}}{q^{4}} \frac{1}{2 m V_{0 \|}} \int_{0}^{2 \pi} \frac{d \varphi}{2 \pi} \bar{\sigma}_{\|}(\varphi) .
$$

It should be emphasized that for ion-electron collision the phase dependent transversal relative velocity $v_{0 \perp}(\varphi)$ is replaced by the electron transversal velocity $v_{e \perp}$ and the relative cyclotron radius $v_{0 \perp} / \omega_{c}$ for identical particles is replaced by $v_{e \perp} / \omega_{c}$. Thus the integrands in Eqs. (29) and (39) (and similarly in other angular-averaged quantities) do not depend on the phase $\varphi(\mathcal{E}(\varphi, s)=\mathcal{E}(s))$ and, therefore, we have $-\left\langle\Delta E_{1}^{(2)}\right\rangle=-\mathcal{E}(s) \rightarrow\left\langle\Delta E_{i}^{(2)}\right\rangle$ for the second order energy transfer to the ion $\left\langle\Delta E_{i}^{(2)}\right\rangle$.

\section{Second order relative energy transfers}

We now turn back to the case of identical particles and consider the evaluation of the angular-averaged relative energy transfer in longitudinal direction, $\left\langle\Delta E_{r \|}^{(2)}\right\rangle=-\left\langle\Delta E_{r \perp}^{(2)}\right\rangle$. According to Eq. (15) the longitudinal relative energy transfer $\left\langle\Delta E_{r \|}^{(2)}\right\rangle$ involves both $\left\langle\Delta v_{\|}^{(2)}\right\rangle$ (37) and the the angular-averaged value of $\left(\Delta v_{\|}^{(1)}\right)^{2}$. The latter is calculated from Eq. (28). The angular-averaging procedure is the same as for deriving Eqs. (29) and (30) and yields

$$
\left\langle\left(\Delta v_{\|}^{(1)}\right)^{2}\right\rangle=\int_{0}^{2 \pi} \frac{d \varphi}{2 \pi} \mathcal{U}_{2}(\varphi, s)
$$

with

$$
\begin{aligned}
\mathcal{U}_{2}(\varphi, s)= & \frac{4(2 \pi)^{2} q^{4} \phi^{4}}{m^{2}\left|v_{r \|}\right|} \int d \mathbf{k} d \mathbf{k}^{\prime} U(\mathbf{k}) U\left(\mathbf{k}^{\prime}\right) k_{\|}^{2} J_{0}(Q s) \delta\left(k_{\|}^{\prime}+k_{\|}\right) \\
& \times \sum_{n=-\infty}^{\infty}(-1)^{n} e^{i n \varsigma\left(\theta-\theta^{\prime}\right)} J_{n}\left(k_{\perp} a\right) J_{n}\left(k_{\perp}^{\prime} a\right) \delta\left(\zeta_{n}(\mathbf{k})\right) .
\end{aligned}
$$

The relative energy change in the longitudinal direction is then calculated using Eqs. (15), (37) and (40)

$$
\left\langle\Delta E_{r \perp}^{(2)}\right\rangle \equiv-\left\langle\Delta E_{r \|}^{(2)}\right\rangle=\int_{0}^{2 \pi} \frac{d \varphi}{2 \pi} \mathcal{E}_{r \perp}(\varphi, s)
$$

with

$$
\mathcal{E}_{r \perp}(\varphi, s) \equiv-\mathcal{E}_{r \|}(\varphi, s)=-\frac{m}{4}\left[\mathcal{U}_{2}(\varphi, s)+2 v_{r \|} \mathcal{U}_{1}(\varphi, s)\right]
$$

For further applications (see Sec. V) also the angular averaged square of the first order relative energy transfer in the transversal direction, $\left\langle\left(\Delta E_{r \perp}^{(1)}\right)^{2}\right\rangle$ is needed, which is given by

$$
\left\langle\left(\Delta E_{r \perp}^{(1)}\right)^{2}\right\rangle=\frac{m^{2}}{4} v_{r \|}^{2}\left\langle\left(\Delta v_{\|}^{(1)}\right)^{2}\right\rangle=\frac{m^{2}}{4} v_{r \|}^{2} \int_{0}^{2 \pi} \frac{d \varphi}{2 \pi} \mathcal{U}_{2}(\varphi, s) .
$$

Similarly to the definition and derivation of Eqs. (33) and (34) we define the related cross-sections by

$$
\begin{aligned}
\sigma_{r \perp} & =\int_{0}^{\infty}\left\langle\Delta E_{r \perp}^{(2)}\right\rangle s d s=\int_{0}^{2 \pi} \frac{d \varphi}{2 \pi} \bar{\sigma}_{r \perp}(\varphi) \\
\sigma_{r 1}^{2} & =\int_{0}^{\infty}\left\langle\left(\Delta E_{r \perp}^{(1)}\right)^{2}\right\rangle s d s=\int_{0}^{2 \pi} \frac{d \varphi}{2 \pi} \bar{\sigma}_{r 1}(\varphi)
\end{aligned}
$$


with

$$
\begin{aligned}
\bar{\sigma}_{r \perp}(\varphi)= & \int_{0}^{\infty} \mathcal{E}_{r \perp}(\varphi, s) s d s=\frac{2(2 \pi)^{2} q^{4} \phi^{4}}{m\left|v_{r \|}\right| \omega_{c}} \int_{0}^{\infty} d t \int d \mathbf{k}|U(\mathbf{k})|^{2} k_{\|} J_{0}\left(2 k_{\perp} a \sin \frac{t}{2}\right) \\
& \times\left[\delta t\left(k_{\|}^{2}+k_{\perp}^{2} \frac{\sin t}{t}\right) \sin \left(k_{\|} \delta t\right)-k_{\|} \cos \left(k_{\|} \delta t\right)\right] \\
\bar{\sigma}_{r 1}(\varphi) & =\frac{m^{2}}{4} v_{r \|}^{2} \int_{0}^{\infty} \mathcal{U}_{2}(\varphi, s) s d s \\
& =2(2 \pi)^{2} q^{4} \phi^{4} \delta \int_{0}^{\infty} d t \int d \mathbf{k}|U(\mathbf{k})|^{2} k_{\|}^{2} \cos \left(k_{\|} \delta t\right) J_{0}\left(2 k_{\perp} a \sin \frac{t}{2}\right)
\end{aligned}
$$

\section{THE ENERGY TRANSFER FOR THE SCREENED AND REGULARIZED POTENTIAL}

For the Coulomb interaction $U(k)=U_{\mathrm{C}}(k)$, the full $2 \mathrm{D}$ integration over the s-space results in a logarithmic divergence of the k-integration in Eqs. (35), (36), (47) and (48). To cure this cutoff parameters $k_{\min }$ and $k_{\max }$ must be introduced, see [14, 20] for details. But the averaged energy transfer, Eq. (29) with Eq. (30), can be evaluated without further approximation for any axially symmetric interaction potential, $U(\mathbf{k})=U\left(\left|k_{\|}\right|, k_{\perp}\right)$. In this case the averaged energy transfer can be represented as the sum of all cyclotron harmonics as it has been done for ion-electron interaction in Ref. [14].

To continue the interaction must be specified. In the following we consider throughout the regularized screened potential $U(\mathbf{r})=U_{\mathrm{R}}(\mathbf{r})$ introduced in Sec. ПA with

$$
U_{\mathrm{R}}(\mathbf{r})=\left(1-e^{-r / \star}\right) \frac{e^{-r / \lambda}}{r}, \quad U_{\mathrm{R}}\left(k_{\|}, k_{\perp}\right)=\frac{2}{(2 \pi)^{2}}\left(\frac{1}{k_{\perp}^{2}+\kappa^{2}}-\frac{1}{k_{\perp}^{2}+\chi^{2}}\right),
$$

where $\kappa^{2}=k_{\|}^{2}+\lambda^{-2}, \chi^{2}=k_{\|}^{2}+d^{-2} a, d^{-1}=\lambda^{-1}+\lambda^{-1}$, for which the $\mathbf{k}$-integrations involved in Eqs. (35), (36), (477) and (48) converge.

\section{A. Second order energy transfer}

A simple but important particular case is that of vanishing cyclotron radius $\left(v_{01 \perp}=v_{02 \perp}=0\right)$, i.e. when initially the electrons move along the magnetic field. From Eqs. (28), (40) and (41), and using the regularized potential (49), it is straightforward to show that in this case $\Delta v_{\|}^{(1)}=0$ and $\mathcal{U}_{2}(\varphi, s)=0$. Substituting Eq. (49) into Eqs. (30), (38) and (43) and setting $a=0$ we obtain after $\mathbf{k}, \mathbf{k}^{\prime}$-integrations

$$
\mathcal{E}(\varphi, s)=-\frac{V_{0 \|}}{v_{r \|}} \mathcal{E}_{r \perp}(\varphi, s)=-\frac{4 q^{4} \phi^{4} V_{0 \|}}{m v_{r \|}^{3} s^{2}}\left[\left(\kappa_{1} s\right) K_{1}\left(\kappa_{1} s\right)-\left(\chi_{1} s\right) K_{1}\left(\chi_{1} s\right)\right]^{2},
$$

where $\kappa_{1}^{2}=\delta^{-2}+\lambda^{-2}, \chi_{1}^{2}=\delta^{-2}+d^{-2}$ and $K_{n}$ are the modified Bessel functions. From Eq. (50) it is seen that the modified Bessel functions guarantee the convergence of the impact parameter integrated energy transfer at small $s$.

Eq. (50) is the leading term of the expansion of the the energy transfers with respect to small cyclotron radius $a$ and was obtained in the limit $a \rightarrow 0$ where the particles move initially along their guiding center trajectories. Moreover, for $\omega_{c} \rightarrow \infty$ also the relative pitch $\delta \rightarrow 0$ and these trajectories are rectilinear along the lines of the magnetic field. The particles just pass each other along a straight line and for symmetry reasons the velocity and energy transfers vanish. For a finite $\omega_{c}$ corresponding to a finite pitch the contribution of the leading term in Eq. (50) describes the perturbation of the guiding centers trajectories. By the reason of symmetry the next order term must be quadratic $\sim a^{2}$ and accounts for the finite relative cyclotron motion of the particles.

As we discussed in Sec. IIIB the energy transfer (29) must be integrated with respect to the impact parameters $s$ for practical applications. For general interaction potential this has been done in Sec. IIIB, Eqs. (33)-(36). In general for a study of the convergence of the $s$-integrated energy transfers we note that the case with $s=a$ is most critical for the convergence of the cross-sections. This is intuitively clear as the gyrating particles at $\left|a_{1}-a_{2}\right|<s<a_{1}+a_{2}$ may hit each other on such a trajectory. This should not matter for the potential (49), which has been regularized 
near the origin for exactly that purpose. On the other hand, the energy transfer for the un-regularized potentials $U_{\mathrm{C}}$ and $U_{\mathrm{D}}$ diverges for $s=a$ (see Ref. [14] for some explicit examples).

For the present case of the regularized and screened interaction potential Eq. (49), i.e. substituting this potential into Eqs. (35) and (36), the impact parameter integrated cross sections are

$$
\begin{aligned}
\bar{\sigma}_{\|}(\varphi)= & -\frac{2 q^{4} \phi^{4} V_{0 \|}}{m \omega_{c}^{3} \lambda^{3}} \frac{\left|v_{r \|}\right|}{v_{r \|}} \int_{0}^{\infty} \frac{t^{2} d t}{R^{3}(t)}\left\{e^{-R(t)}\left[\mathcal{F}_{1}(R(t), \xi t, t)+\frac{4}{\varkappa^{2}-1} \mathcal{F}_{2}(R(t), \xi t, t)\right]\right. \\
& \left.+e^{-\varkappa R(t)}\left[\mathcal{F}_{1}(\varkappa R(t), \varkappa \xi t, t)-\frac{4 \varkappa^{2}}{\varkappa^{2}-1} \mathcal{F}_{2}(\varkappa R(t), \varkappa \xi t, t)\right]\right\}, \\
\bar{\sigma}_{\perp}(\varphi)= & \frac{q^{4} \phi^{4}}{m \omega_{c}^{3} \lambda^{3}} \frac{v_{02 \perp}^{2}-v_{01 \perp}^{2}}{\left|v_{r \|}\right|} \int_{0}^{\infty} \frac{t \sin t d t}{R^{3}(t)}\left\{e^{-R(t)}\left[\mathcal{F}_{3}(R(t), \xi t, t)+\frac{4}{\varkappa^{2}-1} \mathcal{F}_{4}(R(t), \xi t, t)\right]\right. \\
& \left.+e^{-\varkappa R(t)}\left[\mathcal{F}_{3}(\varkappa R(t), \varkappa \xi t, t)-\frac{4 \varkappa^{2}}{\varkappa^{2}-1} \mathcal{F}_{4}(\varkappa R(t), \varkappa \xi t, t)\right]\right\} .
\end{aligned}
$$

Here $\xi=\delta / \lambda=\left|v_{r}\right| \mid / \omega_{c} \lambda, R^{2}(t)=\xi^{2} t^{2}+4\left(a^{2} / \lambda^{2}\right) \sin ^{2}(t / 2), \varkappa=\lambda / d=1+\lambda / \lambda$, and

$$
\begin{gathered}
\mathcal{F}_{1}(R, \zeta, t)=2+2 R-R^{2}+\left(1-\frac{\sin t}{t}\right)\left[R^{2}+R+1-\frac{\zeta^{2}}{R^{2}}\left(R^{2}+3 R+3\right)\right] \\
\mathcal{F}_{2}(R, \zeta, t)=R+1-\frac{1}{R^{2}}\left(1-\frac{\sin t}{t}\right)\left[R^{3}+4 R^{2}+9 R+9-\frac{\zeta^{2}}{R^{2}}\left(R^{3}+6 R^{2}+15 R+15\right)\right], \\
\mathcal{F}_{3}(R, \zeta, t)=2+2 R-R^{2}+\left(1-\frac{\sin t}{t}\right)\left[R^{2}-R-1-\frac{\zeta^{2}}{R^{2}}\left(R^{2}+3 R+3\right)\right] \\
\mathcal{F}_{4}(R, \zeta, t)=R+1-\frac{1}{R^{2}}\left(1-\frac{\sin t}{t}\right)\left[R^{3}+2 R^{2}+3 R+3-\frac{\zeta^{2}}{R^{2}}\left(R^{3}+6 R^{2}+15 R+15\right)\right] .
\end{gathered}
$$

Note that the transversal cross-section $\bar{\sigma}_{\perp}(\varphi)$ vanish at $a_{1}=a_{2}$ and the energy transfer occurs only due to the cm motion along the magnetic field $\bar{\sigma}_{\|}(\varphi)$. We also note in particular the dependence of the sign of the transversal cross-section Eq. (52) on $a_{1}-a_{2}$.

As already discussed in Sec. IIIB the energy transfer in the case of electron-heavy ion collisions is given by the $s$-integrated $\left\langle\Delta v_{\|}^{(2)}\right\rangle$, see Eq. (39), now with $\bar{\sigma}_{\|}(\varphi)$ from Eq. (51).

\section{B. Second order relative energy transfers}

Similarly, substituting Eq. (49) into Eqs. (47) and (48), we obtain for the relative cross-sections $\bar{\sigma}_{r \perp}(\varphi)$ and $\bar{\sigma}_{r 1}(\varphi)$

$$
\begin{gathered}
\bar{\sigma}_{r \perp}(\varphi)=\frac{2 q^{4} \phi^{4}}{m\left|v_{r \|}\right| \omega_{c} \lambda} \int_{0}^{\infty} \frac{d t}{R^{3}(t)}\left\{e^{-R(t)}\left[\mathcal{Y}_{1}(R(t), \xi t, t)+\frac{4}{\varkappa^{2}-1} \mathcal{Y}_{2}(R(t), \xi t, t)\right]\right. \\
\left.+\frac{1}{\varkappa^{2}} e^{-\varkappa R(t)}\left[\mathcal{Y}_{1}(\varkappa R(t), \varkappa \xi t, t)-\frac{4 \varkappa^{2}}{\varkappa^{2}-1} \mathcal{Y}_{2}(\varkappa R(t), \varkappa \xi t, t)\right]\right\}, \\
\bar{\sigma}_{r 1}(\varphi)=2 q^{4} \phi^{4} \xi \int_{0}^{\infty} \frac{d t}{R(t)}\left\{e^{-R(t)}\left[\mathcal{P}_{1}(R(t), \xi t)-\frac{4}{\varkappa^{2}-1} \mathcal{P}_{2}(R(t), \xi t)\right]\right. \\
\left.+e^{-\varkappa R(t)}\left[\mathcal{P}_{1}(\varkappa R(t), \varkappa \xi t)+\frac{4 \varkappa^{2}}{\varkappa^{2}-1} \mathcal{P}_{2}(\varkappa R(t), \varkappa \xi t)\right]\right\}
\end{gathered}
$$

where

$$
\mathcal{P}_{1}(R, \zeta)=1-\frac{\zeta^{2}}{R^{2}}(R+1), \quad \mathcal{P}_{2}(R, \zeta)=\frac{1}{R^{2}}\left[R+1-\frac{\zeta^{2}}{R^{2}}\left(R^{2}+3 R+3\right)\right]
$$




$$
\begin{aligned}
\mathcal{Y}_{1}(R, \zeta, t)= & -R^{2}+\zeta^{2}\left(3 R+3-R^{2}\right)+\zeta^{2}\left(1-\frac{\sin t}{t}\right)\left[R^{2}+R+1-\frac{\zeta^{2}}{R^{2}}\left(R^{2}+3 R+3\right)\right] \\
\mathcal{Y}_{2}(R, \zeta, t)= & \left(\zeta^{2}+1\right)(R+1)-\frac{\zeta^{2}}{R^{2}}\left(R^{2}+3 R+3\right) \\
& -\frac{\zeta^{2}}{R^{2}}\left(1-\frac{\sin t}{t}\right)\left[R^{3}+4 R^{2}+9 R+9-\frac{\zeta^{2}}{R^{2}}\left(R^{3}+6 R^{2}+15 R+15\right)\right] .
\end{aligned}
$$

It must be emphasized that in contrast to the quantity $\bar{\sigma}(\varphi)$ the relative cross-sections $\bar{\sigma}_{r \perp}(\varphi)$ and $\bar{\sigma}_{r 1}(\varphi)$ do not depend on the cm velocity components $V_{0 \|}$ and $V_{0 \perp}$.

Next we also consider the cross-sections $\bar{\sigma}(\varphi), \bar{\sigma}_{r \perp}(\varphi)$ and $\bar{\sigma}_{r 1}(\varphi)$ for vanishing cyclotron radius, $a \rightarrow 0$. In this limit $\bar{\sigma}_{\perp}(\varphi)=0$ and $\bar{\sigma}_{r 1}(\varphi)=0$ as can be easily seen from Eq. (48) as well as by explicitly evaluating Eq. (58) with $R(t)=\xi t$. The integration of Eq. (50) with respect to the impact parameter (see Appendix $\mathrm{A}$ for details) yields

$$
\bar{\sigma}(\varphi)=-\frac{V_{0 \|}}{v_{r \|}} \bar{\sigma}_{r \perp}(\varphi)=-\frac{4 q^{4} \phi^{4} V_{0 \|}}{m v_{r \|}^{3}}\left[\frac{\xi^{2}\left(\varkappa^{2}+1\right)+2}{2 \xi^{2}\left(\varkappa^{2}-1\right)} \ln \frac{\xi^{2} \varkappa^{2}+1}{\xi^{2}+1}-1\right] .
$$

Note that $\bar{\sigma}(\varphi)$ at $a \rightarrow 0$ can be alternatively evaluated from Eq. (51). In this limit the expression for $R(t)$ is simplified to $R(t)=\xi t$. After performing the $t$-integration we again arrive at Eq. (62). In addition Eq. (62) is approximately valid also for finite cyclotron radius $a$, assuming that the longitudinal velocity $v_{r \|}$ is larger than the transversal ones, $v_{0 \perp}$ and $V_{0 \perp}$. Indeed, in this case $R(t) \simeq \xi t$ since $\delta \gg a$ and in Eqs. (51) and (52) the transversal cross-section $\bar{\sigma}_{\perp}(\varphi)$ can be neglected compared to the longitudinal one. This indicates that in the high velocity limit with $v_{r \|} \gg v_{0 \perp}, V_{0 \perp}$ the transversal motion of the particles as well as its cm transversal motion are not important and can be neglected. This high velocity limit of the cross-section $\bar{\sigma}_{r 1}(\varphi)$ is obtained from Eq. (58). Since only the contribution of small $t$ is important the function $R(t)$ is approximated by $R(t) \simeq t\left(\xi^{2}+a^{2} / \lambda^{2}\right)^{1 / 2} \simeq \xi t\left(1+a^{2} / 2 \delta^{2}\right)$ (here we keep the small term $\sim a^{2} / \delta^{2}$ because $\bar{\sigma}_{r 1}(\varphi)$ vanishes at $a \rightarrow 0$ ). Using this result for $R(t)$ from Eqs. (52), (58) and (62) in the high velocity limit we obtain within the leading term approximation for the cross-sections

$$
\begin{aligned}
& \bar{\sigma}_{r 1}(\varphi) \simeq 2 q^{4} \phi^{4} \frac{v_{0 \perp}^{2}}{v_{r \|}^{2}} \Lambda(\varkappa), \\
& \bar{\sigma}_{\|}(\varphi) \simeq-\frac{V_{0 \|}}{v_{r \|}} \bar{\sigma}_{r \perp}(\varphi) \simeq-\frac{4 q^{4} \phi^{4} V_{0 \|}}{m v_{r \|}^{3}} \Lambda(\varkappa), \\
& \bar{\sigma}_{\perp}(\varphi) \simeq \frac{2 q^{4} \phi^{4}}{m v_{r \|}^{4}}\left(v_{02 \perp}^{2}-v_{01 \perp}^{2}\right) \Lambda(\varkappa) .
\end{aligned}
$$

Here $\Lambda(\varkappa)$ is a generalized Coulomb logarithm and is given by Eq. (A5). Note that $\bar{\sigma}(\varphi)$ and $\bar{\sigma}_{r \perp}(\varphi)$ are isotropic, i.e. do not depend on $\varphi$ while $\bar{\sigma}_{r 1}(\varphi)$ contains a term which is proportional to $\cos \varphi$. Also, in the high velocity limit the cross-sections do not depend on the magnetic field strength and decays as $\bar{\sigma}_{\|}(\varphi) \sim v_{r \|}^{-3}, \bar{\sigma}_{\perp}(\varphi) \sim v_{r \|}^{-4}, \bar{\sigma}_{r \perp}(\varphi) \sim v_{r \|}^{-2}$ and $\bar{\sigma}_{r 1}(\varphi) \sim v_{r \|}^{-2}$.

Finally we briefly turn to the case of small relative velocity, $v_{r \|} \ll v_{0 \perp}, V_{0 \perp}$. It should be emphasized that the integral representations of the cross-sections, Eqs. (51), (57) and (58), are not adopted for evaluation of these quantities at small velocities. For this purpose it is much more convenient to use an alternative Bessel-function representation of the cross-sections as shown in Appendix B. In addition, it is expected that the limit of small $v_{r}$ is the most critical regime for a violation of the perturbation theory employed here. Therefore explicit analytical expressions in this limit can be useful for an improvement of the perturbation theory by comparing the analytical results with numerical simulations, see Sec. VI.

\section{IMPROVED TREATMENT FOR THE REPULSIVE INTERACTION}

The magnetic field drastically changes the scattering problem of two charged particles as discussed in Sec. I One important consequence of a strong magnetic field is the backscattering of the particles from the repulsive potential barrier even at a finite impact parameter $s$ (let us recall that in the case of Rutherford scattering this occurs at either vanishing impact parameters, $s \rightarrow 0$, or at vanishing relative velocity). For instance, in a strong magnetic field with $a \ll \lambda, s$ and in the case of attractive interaction the velocity and energy transfers are very small and for symmetry reasons vanish with increasing $B$, see the similar discussion in Sec. IV] However, in the case of repulsive interaction the magnetic field together with the interaction potential forms a potential barrier because of the particles 
motion is effectively one-dimensional. Here two possible scattering regimes must be clearly distinguished. To this end, we consider an exactly solvable model for two interacting particles moving in the presence of an infinitely strong magnetic field on rectilinear trajectories along the field with vanishing cyclotron radii. Introducing the relative coordinate $\zeta=z_{1}(t)-z_{2}(t)$ the relative energy conservation (note that the $\mathrm{cm}$ energy is also conserved since $\mathbf{V}=$ $\mathbf{b} V_{\|}(t)=\mathbf{b} V_{0 \|}=$ const) for the regularized Yukawa potential can be written in the form:

$$
\dot{\zeta}^{2}=v_{r \|}^{2}\left[1-\varsigma_{1} \varsigma_{2} \frac{s_{0}}{r}\left(1-e^{-r / \star}\right) e^{-r / \lambda}\right],
$$

where $\varsigma_{1}=\left|q_{1}\right| / q_{1}, \varsigma_{2}=\left|q_{2}\right| / q_{2}, r^{2}(t)=\zeta^{2}(t)+s^{2}, s_{0}=2\left|q_{1} q_{2}\right| \phi^{2} / \mu v_{r \|}^{2}$. Here $\mu$ is the reduced mass, $s$ is the impact parameter and $v_{r \|}$ is the initial longitudinal relative velocity of the particles. Since the relative energy is conserved there is no relative energy transfer and $\Delta E_{r}=0$. Then the energy transfer of the particle 1 is related to the relative velocity transfer $\Delta v_{\|}=\dot{\zeta}(+\infty)-\dot{\zeta}(-\infty)$ by $\Delta E_{1}=\mu V_{0 \|} \Delta v_{\|}$, see Eq. (14). For reasons of symmetry, no velocity can be transferred from particle 2 to the particle 1 if the interaction is attractive $\left(q_{1} q_{2}<0\right)$, see, e.g., Eq. (66). This may also be true for repulsive case $\left(q_{1} q_{2}>0\right)$. For instance, at $s_{0}<\lambda$ (or equivalently $x=v_{r \|} / v_{s}>(2 / \nu)^{1 / 2}$ with $\nu=\lambda / \lambda$ and $v_{s}^{2}=\left|q_{1} q_{2}\right| \phi^{2} / \mu \lambda$ ) and arbitrary $s$ as well as at $s_{0}>\lambda$ (or equivalently $x<(2 / \nu)^{1 / 2}$ ) and $s>s_{m}=\lambda \eta_{m}(x)$, where $\eta_{m}(x)$ is the root of the transcendental equation

$$
\frac{1}{\eta_{m}}\left(1-e^{-\eta_{m} / \nu}\right) e^{-\eta_{m}}=\frac{x^{2}}{2}
$$

and the energy of two particles relative motion is larger than the energy of the potential barrier which again yields vanishing velocity and energy transfers for repulsive interaction. Thus for $q_{1} q_{2}>0$ the energy transfer occurs at $s_{0}>\lambda$ and $s<s_{m}$. In this case the velocity transfer is $\Delta v_{\|}=-2 v_{r \|}$ which corresponds to a reversion of the initial motion, i.e. to a backscattering event. Then the energy transfer is

$$
\Delta E_{1}=-\Delta E_{2}=-2 \mu V_{0 \|} v_{r \|} \Theta\left(q_{1} q_{2}\right) \Theta\left(v_{c}^{2}-v_{r \|}^{2}\right) \Theta\left(s_{m}-s\right),
$$

where $\Theta(z)$ is the Heavyside function and $v_{c}^{2}=2\left|q_{1} q_{2}\right| \notin^{2} / \mu \lambda$.

Consider now the energy-velocity transfers integrated with respect to the impact parameters $s$. The result reads

$$
-\frac{1}{\lambda^{2}} \int_{0}^{\infty} \frac{\Delta E_{1}(s)}{\mu V_{0 \|} v_{s}} s d s=-\frac{1}{\lambda^{2}} \int_{0}^{\infty} \frac{\Delta v_{\|}(s)}{v_{s}} s d s=x \eta_{m}^{2}(x) .
$$

Here $\eta_{m}$ is a function of $x=v_{r \|} / v_{s}$. (Note that this function vanishes at $x>(2 / \nu)^{1 / 2}$, where the transcendental equation (67) has no solution). Consider two limiting cases. At $v_{r \|} / v_{s} \lesssim(2 / \nu)^{1 / 2}$ we obtain from Eq. (67)

$$
\eta_{m}(x) \simeq \frac{(2 \nu)^{3 / 2}}{2 \nu+1}\left[\left(\frac{2}{\nu}\right)^{1 / 2}-x\right]
$$

and the $s$-integrated velocity transfer vanishes as $\sim\left[(2 / \nu)^{1 / 2}-x\right]^{2}$. At $v_{r \|} / v_{s} \rightarrow 0$, Eq. (67) yields

$$
\eta_{m}(x) \simeq \ln \left[\frac{2 / x^{2}}{\ln \left(2 / x^{2}\right)}\right] .
$$

and the $s$-integrated velocity transfer vanishes like

$$
-\frac{1}{\lambda^{2}} \int_{0}^{\infty} \frac{\Delta v_{\|}(s)}{v_{s}} s d s \simeq x \ln ^{2}\left[\frac{2 / x^{2}}{\ln \left(2 / x^{2}\right)}\right] \rightarrow 0 .
$$

From this simple example it is clear that the perturbative treatment developed in the previous sections is not applicable for repulsive interaction and in the presence of strong magnetic field when the hard collisions like backscattering events may occur. Nevertheless the second order treatment for the repulsive case can be improved when the energy transfers due to the hard collisions are involved in the theory as the leading terms. The simple example considered above suggests that this can be done by explicitly using the energy conservation, see, e.g. Eq. (66). For simplicity consider the case when the relative energy of particles is conserved, $\Delta E_{r}=0$. Then from Eq. (15) (in the general case $m / 2$ must be replaced here by the reduced mass $\mu$ ) and the obvious relation $\Delta E_{r \|}=-\Delta E_{r \perp}$ we obtain a quadratic equation for the relative velocity transfer $\Delta v_{\|}$which has two real solutions

$$
\Delta v_{\|}^{\mathrm{I}}=-v_{r \|}(1-\sqrt{1-\phi}), \quad \Delta v_{\|}^{\mathrm{II}}=-v_{r \|}(1+\sqrt{1-\phi})
$$


with $\phi=2 \Delta E_{r \perp} / \mu v_{r \|}^{2}$. The velocity transfers $\Delta v_{\|}^{\mathrm{I}}$ and $\Delta v_{\|}^{\mathrm{II}}$ correspond to two different scattering regimes. In particular, in the limit of strong magnetic field the transversal energy transfer is small, $\phi \ll 1$, because of the transversal motion is strongly hindered and

$$
\Delta v_{\|}^{\mathrm{I}} \simeq-v_{r \|} \Phi, \quad \Delta v_{\|}^{\mathrm{II}} \simeq-v_{r \|}(2-\Phi),
$$

where $\Phi=\frac{1}{2} \phi+\frac{1}{8} \phi^{2}$. In the limit of vanishing $\phi, \Delta v_{\|}^{\mathrm{I}} \rightarrow 0$ while $\Delta v_{\|}^{\mathrm{II}} \rightarrow-2 v_{r \|}$ which correspond to over the barrier and backscattering events, respectively. Thus, in contrast to the Rutherford classical scattering the magnetic field may form two scattering channels, I and II. For instance, the scattering with an attractive interaction is realized in the regime I while in the repulsive case the scattering may occur in both regimes I and II depending on the relation between the initial relative energy, the height of the potential barrier and the strength of the magnetic field. In the case of the infinitely strong magnetic field the boundary between I and II is fixed by the arguments of the Heavyside functions in Eq. (68). According to Eq. (73) the velocity transfers $\Delta v_{\|}^{\mathrm{I}}$ and $\Delta v_{\|}^{\mathrm{II}}$ obey an exact relation $\Delta v_{\|}^{\mathrm{I}}+\Delta v_{\|}^{\mathrm{II}}=-2 v_{r \|}$.

We further consider the kinematically allowed range for the regimes I and II. The dimensionless transversal energy transfer $\phi$ can range $-\phi_{\max } \leqslant \phi \leqslant 1$, where $\phi_{\max }=v_{0 \perp}^{2} / v_{r \|}^{2}, v_{0 \perp}$ is the initial transversal relative velocity. Here $\phi=-\phi_{\max }(\phi=1)$ corresponds to a complete transfer of the initial relative transversal (longitudinal) motion to the parallel (transversal) one. Therefore the quantities $\Delta v_{\|}^{\mathrm{I}}$ and $\Delta v_{\|}^{\mathrm{II}}$ are restricted in the domains

$$
\begin{gathered}
-v_{r \|} \leqslant \Delta v_{\|}^{\mathrm{I}} \leqslant v_{r \|} \frac{\phi_{\max }}{\sqrt{1+\phi_{\max }}+1}, \\
-v_{r \|}\left(1+\sqrt{1+\phi_{\max }}\right) \leqslant \Delta v_{\|}^{\mathrm{II}} \leqslant-v_{r \|},
\end{gathered}
$$

where the boundary between I and II is fixed by $\Delta v_{\|}=-v_{r \|}$.

The discussion above indicates that the second order perturbative treatment for the repulsive interaction in the regime II can be improved if instead of the standard approximation developed in the previous sections, the second relation in Eq. (74) is used. In particular, in the presence of a strong magnetic field the modified second order velocity transfer reads

$$
\Delta v_{\|}^{(2)} \simeq-2 v_{r \|}+v_{r \|}\left[\frac{\Delta E_{r \perp}^{(2)}}{\mu v_{r \|}^{2}}+\frac{1}{2}\left(\frac{\Delta E_{r \perp}^{(1)}}{\mu v_{r \|}^{2}}\right)^{2}\right] .
$$

Here $\Delta E_{r \perp}^{(1)}$ and $\Delta E_{r \perp}^{(2)}$ are the first and second order relative transversal energy transfers, respectively (see, e.g., Eqs. (42)-(44) for collisions of two identical particles). Note that the second term in Eq. (77) within the square brackets enters here by the opposite sign compared to the standard perturbative treatment where the first (backscattering) term in Eq. (77) is not involved. Since we assumed the conservation of the relative energy of the particles these results are valid both for $\mathrm{BC}$ of two identical particles and for ion-electron collisions (with $v_{i \perp}=0$ ).

\section{COMPARISON WITH SIMULATIONS}

\section{A. Classical trajectory Monte-Carlo (CTMC) simulations}

A fully numerical treatment is required for applications beyond the perturbative regime and for checking the validity of the perturbative approach outlined above. In the present case of binary ion-electron or electron-electron collisions in a magnetic field and with the effective interaction $U_{\mathrm{R}}(r)$ (49) the numerical evaluation of the $\mathrm{BC}$ energy loss is very complicated, but can be successfully investigated by classical trajectory Monte-Carlo (CTMC) simulations [10, 11, 12]. In the CTMC method [25] the trajectories for the relative motion between the ion and an electron are calculated by a numerical integration of the equations of motion (i.e. Eq. (44) for electron-electron collisions and Eq. (9) for ion-electron collisions, respectively), starting with initial conditions for the parallel $v_{r \|}$ and the transverse $\mathbf{v}_{0 \perp}$ relative velocity. The initial positions are chosen to correspond to a certain impact parameter $\mathbf{s}$ and are located outside the interaction zone, which is - employing a screened interaction like (49) - defined as a sphere of several screening lengths $\lambda$ about the ion. The numerical calculation stops after the electron has left this interaction zone, that is, when the collision is completed. Deducing the velocity changes from the initial and final velocities $v_{\|}, \mathbf{v}_{\perp}$, $V_{\|}, \mathbf{V}_{\perp}$ yields the energy transfer $\Delta E_{1}$ (14) (with $\Delta E_{\mathrm{r}}=0$ ) or $\Delta E_{i}$ (16). The required accuracy is achieved by using a modified Velocity-Verlet algorithm which has been specifically designed for particle propagation in a (strong) 

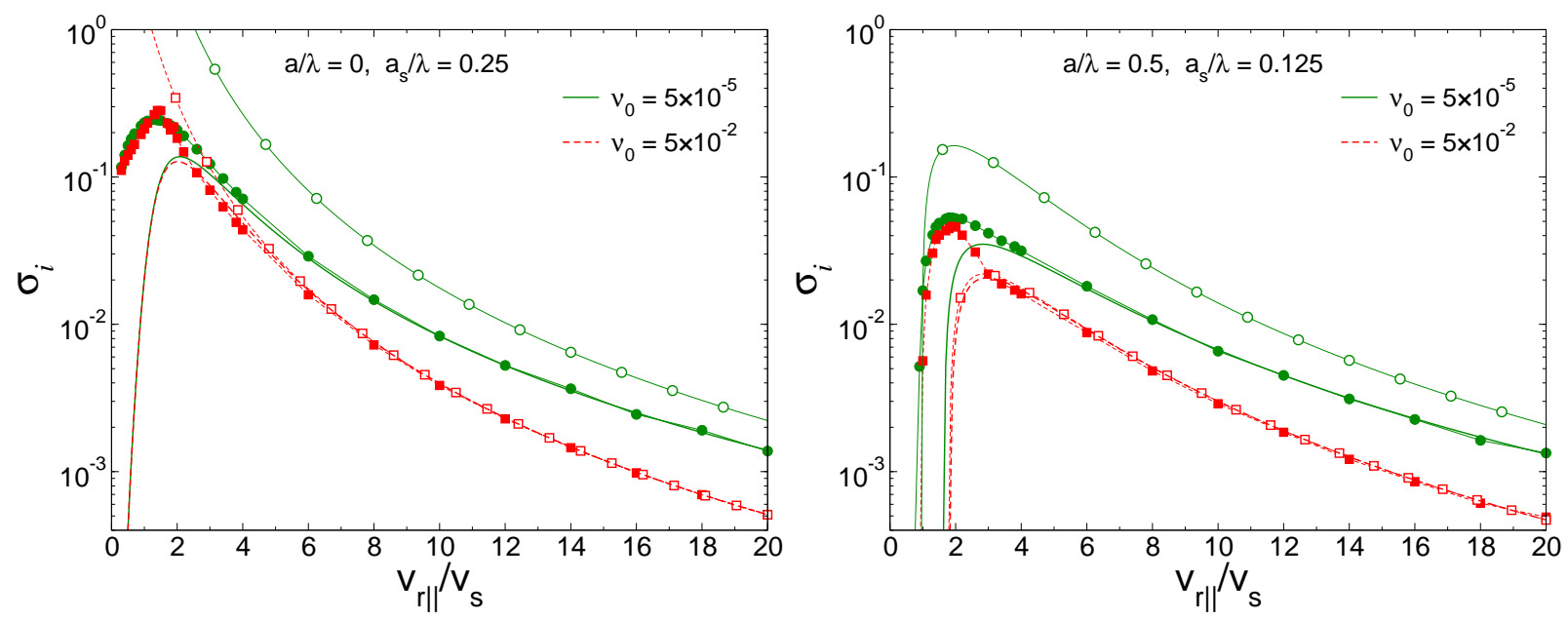

FIG. 1: (Color online) The cross-section $\sigma_{i}$ Eq. (81) for ion-electron (with $Z>0$ ) collisions in terms of the dimensionless relative velocity component $v_{r \|} / v_{s}$ at $a=0, a_{s} / \lambda=0.25$ (left panel) and $a / \lambda=0.5, a_{s} / \lambda=0.125$ (right panel). The solid and dashed curves were obtained for $\nu_{0}=\lambda_{0} / \lambda=5 \times 10^{-5}$ and $\nu_{0}=5 \times 10^{-2}$, respectively. The curves with filled symbols are from CTMC simulations. The curves with open symbols represent the second order perturbative treatment with constant $\lambda=\lambda_{0}$. The results of the second order perturbative treatment with a modified (dynamical) cutoff parameter $\lambda\left(v_{r \|}\right)($ see Eq. (82 $)$ are given by the curves without symbols.

magnetic field [26, 27], and by adapting continuously the actual time-step by monitoring the constant of motion $E_{\mathrm{r}}$ (8) or $K$ (11). The resulting relative deviations of $E_{\mathrm{r}}$ or $K$ are of the order of $10^{-6}-10^{-5}$.

The desired average over the initial phases, i.e. over the orientation of the transverse relative velocity $\mathbf{v}_{0 \perp}$ and the impact parameter $\mathbf{s}$ is performed by a Monte-Carlo sampling [28, 29] of a large number of trajectories with different initial values. The actual number of computed trajectories is adjusted by monitoring the convergence of the averaging procedure. Around $10^{5}-10^{6}$ trajectories are typically needed for the energy transfer for one set of initial relative and $\mathrm{cm}$ velocities and at a given magnetic field.

\section{B. Results}

For the forthcoming discussion we put the equation of the relative motion of two particles in a more appropriate dimensionless form by scaling lengths in units of the screening length $\lambda$ and velocities in units of a characteristic velocity $v_{s}$ defined by

$$
v_{s}^{2}=\frac{\left|q_{1} q_{2}\right| \phi^{2}}{\mu \lambda}
$$

Let us recall that $q_{1}=q_{2}=-1, \mu=m / 2$ and $q_{1}=-1, q_{2}=Z, \mu=m$ for electron-electron and ion-electron collisions, respectively. This velocity $v_{s}$ gives a measure for the strength of the Coulomb interaction with respect to the (initial) kinetic energy of relative motion $\mu v_{r}^{2} / 2$. For $v_{r}<v_{s}$ the kinetic energy is small compared to the characteristic potential energy $\left|q_{1} q_{2}\right| \phi^{2} / \lambda$ in a screened Coulomb potential and we expect to be in a non-perturbative regime. A perturbative treatment on the other hand should be applicable for $v_{r} \gg v_{s}$.

The scaled version of equations (4) or (9) only depends on the two dimensionless parameters $a_{s} / \lambda$ and $\lambda / \lambda$, and the initial conditions with positions scaled in $\lambda$ and velocities in $v_{s}$. Here $a_{s}=v_{s} / \omega_{c}$ is the cyclotron radius for $v_{\perp}=v_{s}$ and the parameter $a_{s} / \lambda \propto v_{s} / B$ represents a measure for the strength of the magnetic field compared to the strength of the Coulomb interaction (which is $\propto v_{s}^{2}$, see (78)). The ratio $\lambda / \lambda$ describes the amount of softening of the screened interaction at $r \rightarrow 0$ with $q_{1} q_{2} \phi^{2} U_{\mathrm{R}}(r \rightarrow 0) \rightarrow q_{1} q_{2} \phi^{2} / \lambda$.

In the analytical perturbative approach we thus apply the same scaling of length and velocities and introduce for electron-electron collisions the dimensionless cross-sections

$$
\begin{gathered}
\sigma_{\|}=-\frac{1}{m V_{0 \|} v_{s} \lambda^{2}} \int_{0}^{2 \pi} \frac{d \varphi}{2 \pi} \bar{\sigma}_{\|}(\varphi), \\
\sigma_{\perp}=-\frac{1}{m v_{s}^{2} \lambda^{2}} \int_{0}^{2 \pi} \frac{d \varphi}{2 \pi} \bar{\sigma}_{\perp}(\varphi) .
\end{gathered}
$$



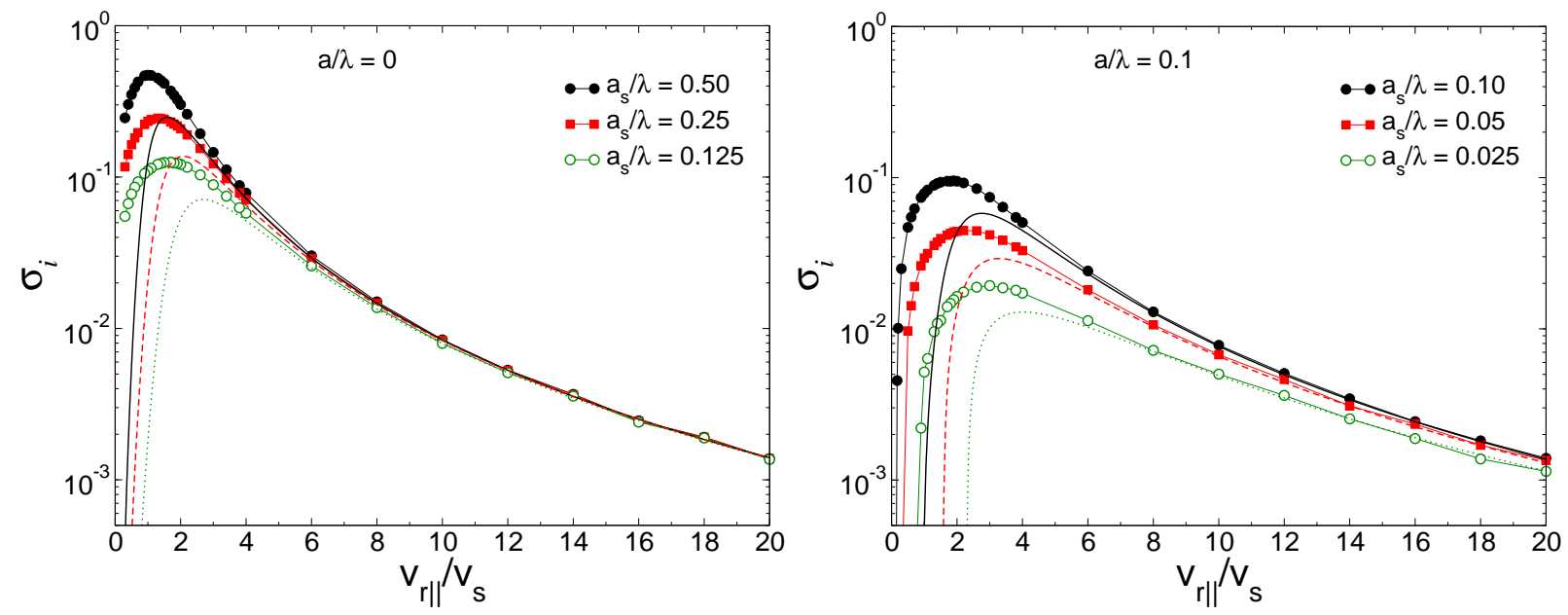

FIG. 2: (Color online) The cross-section $\sigma_{i}$ Eq. (81) for ion-electron (with $Z>0$ ) collision in terms of dimensionless relative velocity component $v_{r \|} / v_{s}$ at $a=0$ (left panel) and $a / \lambda=0.1$ (right panel). The curves with and without symbols correspond to CTMC simulations and the second order perturbative treatment, respectively. Left panel, $a_{s} / \lambda=0.5$ (solid curve), $a_{s} / \lambda=0.25$ (dashed curve), $a_{s} / \lambda=0.125$ (dotted curve). Right panel, $a_{s} / \lambda=0.1$ (solid curve), $a_{s} / \lambda=0.05$ (dashed curve), $a_{s} / \lambda=0.025$ (dotted curve).
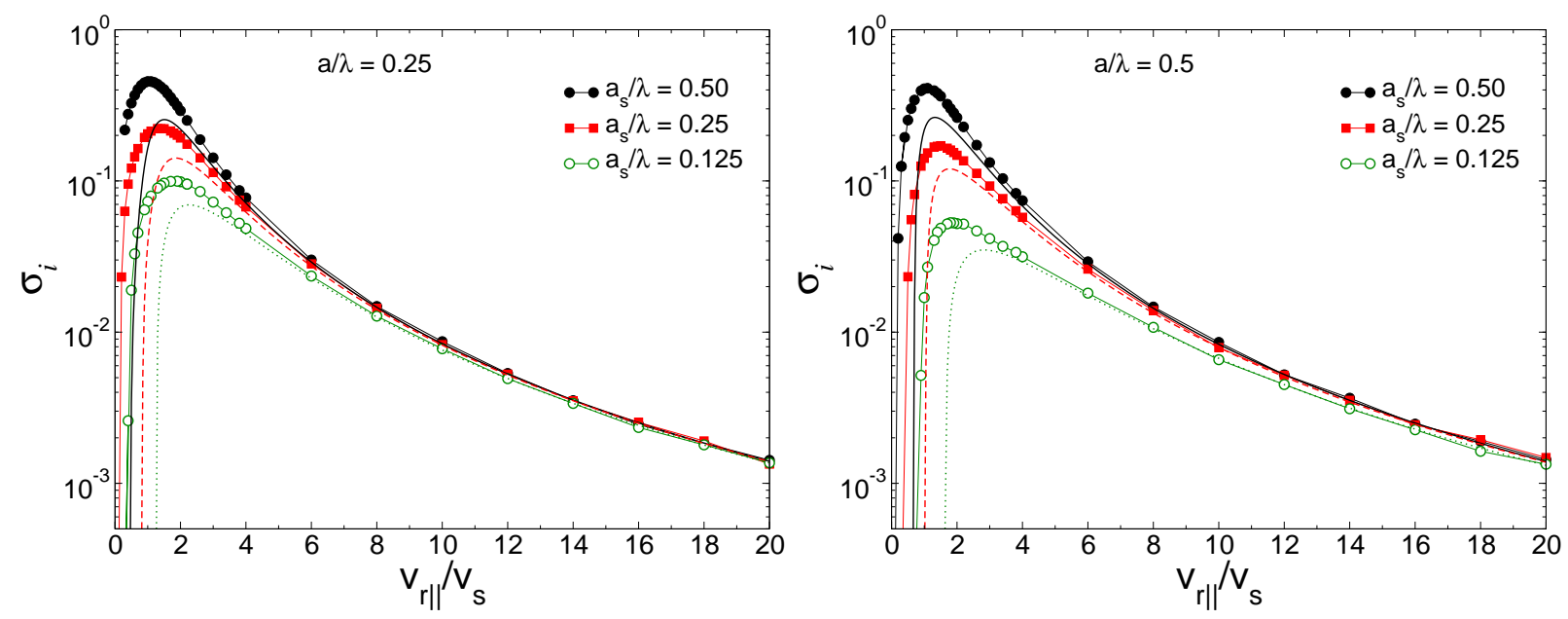

FIG. 3: (Color online) Same as in Fig. 2 but for $a / \lambda=0.25$ (left panel) and $a / \lambda=0.5$ (right panel). In left and right panels, $a_{s} / \lambda=0.5$ (solid), $a_{s} / \lambda=0.25$ (dashed), $a_{s} / \lambda=0.125$ (dotted).

The corresponding dimensionless cross-section for ion-electron interaction is given by

$$
\sigma_{i}=\frac{1}{m v_{i \|} v_{s} \lambda^{2}} \int_{0}^{\infty}\left\langle\Delta E_{i}\right\rangle s d s=-\frac{1}{v_{s} \lambda^{2}} \int_{0}^{\infty}\left\langle\Delta v_{\|}\right\rangle s d s .
$$

It should be noted that in the regimes where the cross-section $\bar{\sigma}_{\|}(\varphi)$ is not strongly sensitive with respect to the initial phases $\varphi$ the quantity $\sigma_{i}$ in Eq. (81) is approximately given by $\sigma_{i} \simeq 2 \sigma_{\|}$(see also the relation (39)) in the units introduced above. An example of such regime has been considered in Sec. IVB where $\bar{\sigma}_{\|}(\varphi)$ in the high-velocity limit and in leading order is independent on $\varphi$, see Eq. (64). One can expect that the approximate relation between the ion-electron and electron-electron cross-sections is valid within second order perturbative treatment with arbitrary $Z$ and within exact CTMC simulations, where, however, the ion-electron interaction must be repulsive $(Z<0)$.

Next we specify the cutoff parameter $\lambda$ which is a measure of softening of the interaction potential at short distances. As we discussed in previous sections the regularization in the potential (49) is sufficient to guarantee the existence of the $s$-integrated energy transfers, see e.g. Eq. (33), but there remains the problem of treating hard collisions. For a perturbation treatment the change in relative velocity must be small compared to $v_{r}$ and this condition is increasingly difficult to fulfill in the regime $v_{r} \rightarrow 0$. This suggests a physically reasonable procedure: The potential must be 

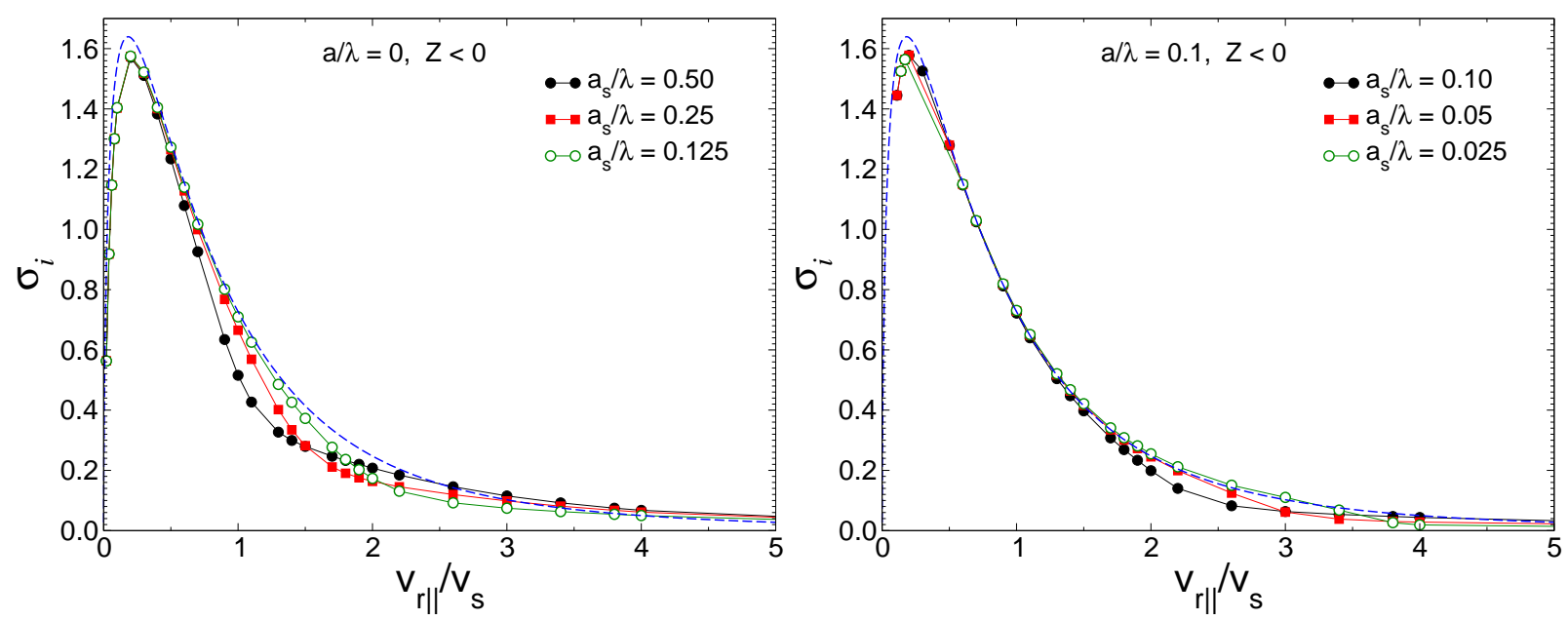

FIG. 4: (Color online) The cross-section $\sigma_{i}$ Eq. (81) for ion-electron collision with $Z<0$ at $a=0$ (left panel) and $a / \lambda=0.1$ (right panel). The curves with symbols correspond to CTMC simulations. Left panel, $a_{s} / \lambda=0.5$ (filled circles), $a_{s} / \lambda=0.25$ (squares), $a_{s} / \lambda=0.125$ (open circles). Right panel, $a_{s} / \lambda=0.1$ (filled circles), $a_{s} / \lambda=0.05$ (squares), $a_{s} / \lambda=0.025$ (open circles). The dashed curves are obtained employing Eq. (69) with constant cutoff parameter $\nu=\nu_{0}=5 \times 10^{-5}$.
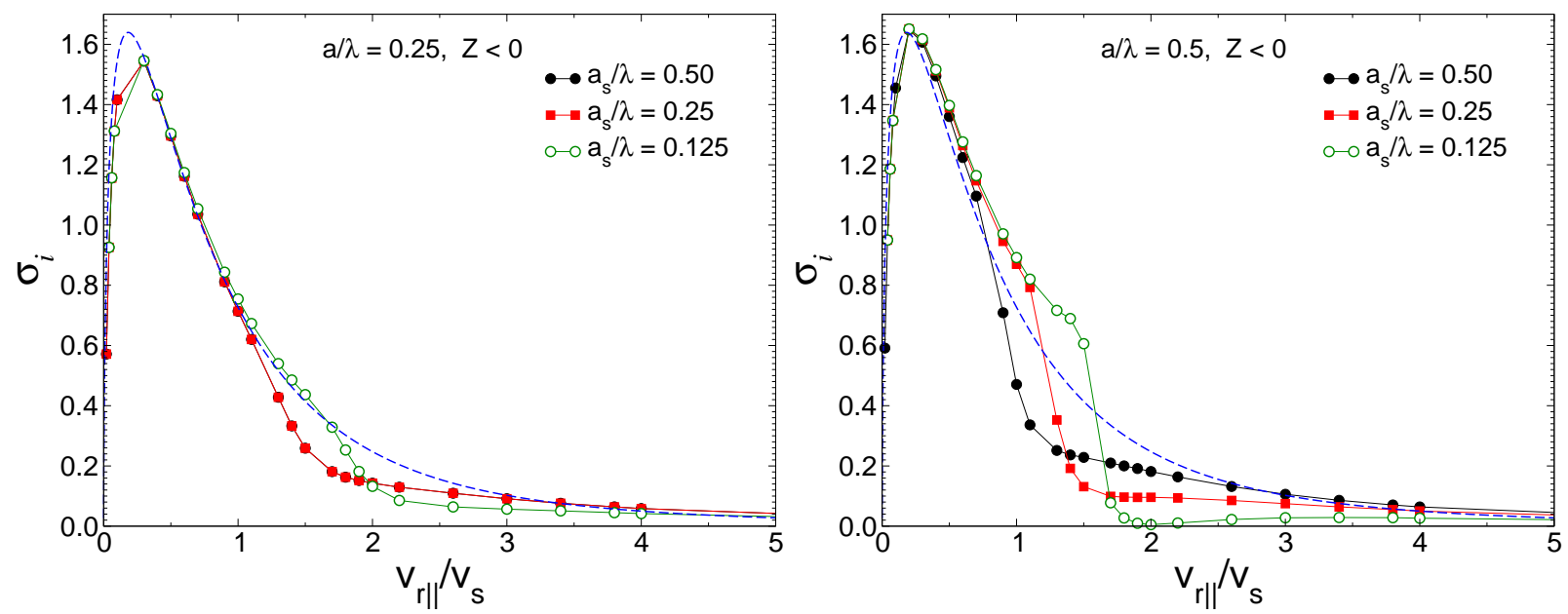

FIG. 5: (Color online) Same as in Fig. 4 but for $a / \lambda=0.25$ (left panel) and $a / \lambda=0.5$ (right panel). In left and right panels, $a_{s} / \lambda=0.5$ (filled circles), $a_{s} / \lambda=0.25$ (squares), $a_{s} / \lambda=0.125$ (open circles).

softened near the origin. In fact the parameter $\lambda$ which describes the effects of quantum diffraction should be related to the de Broglie wavelength which is inversely proportional to $v_{r}$. Here within classical picture of collisions we employ in a perturbative treatment the dynamical cutoff parameter $\varkappa\left(v_{r \|}\right)=1+\lambda / \lambda\left(v_{r \|}\right)$, where

$$
\lambda^{2}\left(v_{r \|}\right)=C b_{0}^{2}\left(v_{r \|}\right)+\lambda_{0}^{2}, \quad b_{0}\left(v_{r \|}\right)=\frac{\left|q_{1} q_{2}\right| \phi^{2}}{\mu\left(v_{r \|}^{2}+v_{0 \perp}^{2}\right)} .
$$

In the case of ion-electron collision $\left|q_{1} q_{2}\right|, \mu$ and $v_{0 \perp}$ in Eq. (82) have to be replaced by $|Z|, m$ and $v_{e \perp}$, respectively. Here $\lambda_{0}$ is some constant cutoff parameter, and $b_{0}\left(v_{r \|}\right)$ is the distance of closest approach of two charged particles in the absence of a magnetic field. Also in Eq. (82) we have introduced an additional fitting parameter $C$. For determining $C$ we consider the second order transport cross-section $\sigma_{\mathrm{tr}}=-\left(2 \pi / \mu v_{r \|} V_{0 \|}\right) \sigma$, where $\sigma$ is given by Eq. (33). For the regularized potential and in high-velocity limit $\sigma$ is given by Eq. (64), where the generalized Coulomb logarithm $\Lambda(\varkappa)$ and the cutoff $\varkappa=\varkappa\left(v_{r \|}\right)$ are determined by Eqs. (A5) and (82), respectively. Setting $\lambda_{0}=0$ the obtained high-velocity transport cross-section is then compared with an exact asymptotic expression derived in Ref. [30] for the Yukawa-type (i.e. with $\lambda \rightarrow 0$ ) interaction potential which yields $C=e^{2 \gamma-1} / 4 \simeq 0.292$, where $\gamma$ is Euler's constant. As will be shown below the second order cross-sections with dynamical cutoff parameter (82) excellently agree with CTMC simulations at high velocities. The CTMC simulations have been carried out with constant $\lambda=\lambda_{0} \ll \lambda$ 

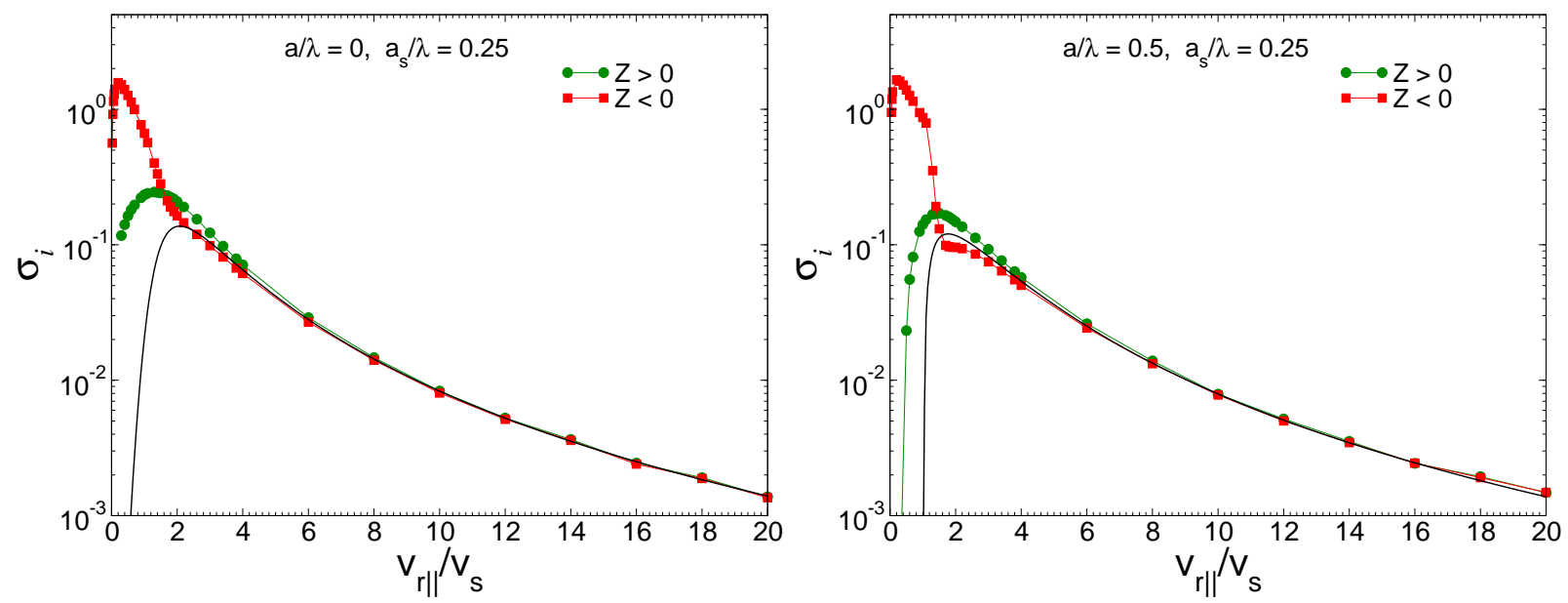

FIG. 6: (Color online) Comparison of the CTMC simulations for the attractive (circles) and repulsive (squares) ion-electron interactions at $a_{s} / \lambda=0.25$. The solid curves correspond to the second order theory with modified cutoff parameter. In left and right panels $a=0$ and $a / \lambda=0.5$, respectively.
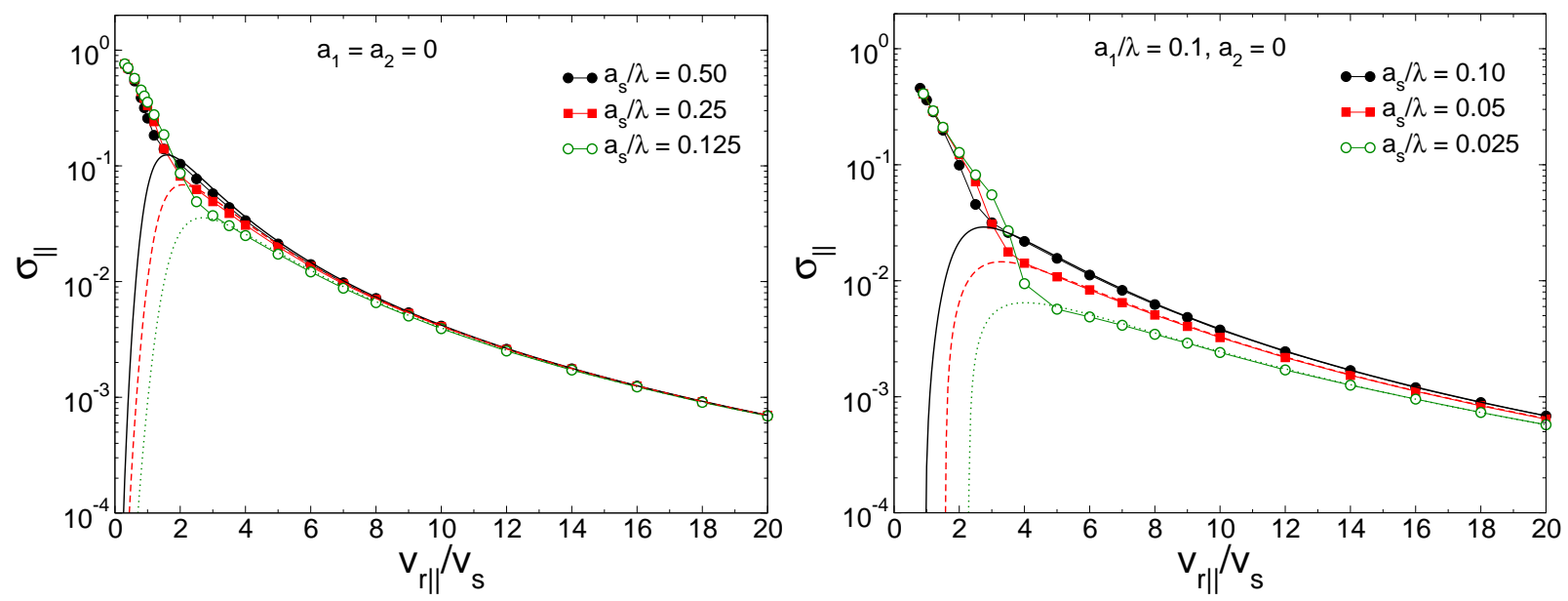

FIG. 7: (Color online) The cross-section $\sigma_{\|}$Eq. (79) for electron-electron collision in terms of dimensionless relative velocity component $v_{r \|} / v_{s}$ for $a_{2}=0$ and at $a_{1}=0$ (left panel) and $a_{1} / \lambda=0.1$ (right panel). The curves with and without symbols correspond to CTMC simulations and the second order perturbative treatment, respectively. Left panel, $a_{s} / \lambda=0.5$ (solid), $a_{s} / \lambda=0.25$ (dashed), $a_{s} / \lambda=0.125$ (dotted). Right panel, $a_{s} / \lambda=0.1$ (solid), $a_{s} / \lambda=0.05$ (dashed), $a_{s} / \lambda=0.025$ (dotted).

(or $\varkappa=\varkappa_{0} \simeq \lambda / \lambda_{0} \gg 1$ ), that is, the interaction is almost Coulomb at short distances. As an example in Fig. 1] we compare the cross-sections $\sigma_{i}$ obtained with CTMC simulations (curves with filled symbols) and within second order perturbative treatment either with constant $\lambda=\lambda_{0}$ (open symbols) or dynamical cutoff parameters 82) (curves without symbols). Two distinct cases are considered here with $\nu_{0}=\lambda_{0} / \lambda=5 \times 10^{-5}$ and $\nu_{0}=5 \times 10^{-2}$. Also $a=0$ (no cyclotron motion at the initial state), $a_{s} / \lambda=0.25$ and $a / \lambda=0.5, a_{s} / \lambda=0.125$ in left and right panels, respectively. It is seen that with respect to the 'softness' of the regularized potential the agreement between CTMC and the perturbative treatment improves, keeping all other parameters fixed, for increasing $\lambda / \lambda$, that is a weaker interaction potential $U_{\mathrm{R}}$.

Systematic investigations and comparisons of the cross-sections determined by the CTMC simulations and the second order perturbative treatment Eqs. (51), (52), (79)-(81) with the dynamical cutoff parameter $\lambda\left(v_{r \|}\right)$ (82) are presented in Figs. 2 11. We have also compared the CTMC results with the simple (exact) model given by Eq. (69) for a repulsive ion-electron interactions, Figs. 4 and 5 . Shown are $\sigma_{i}$ for attractive (with $Z>0$ ) and repulsive (with $Z<0$ ) interactions (Figs. 2 6), $\sigma_{\|}$(Figs. 7 [9), and $\sigma_{\perp}$ (Figs. 10 and11) as functions of $v_{r \|} / v_{s}$ for fixed cyclotron radii $a, a_{1}$ and $a_{2}$ and varying the strength of the magnetic field $a_{s} / \lambda=B_{s} / B$ with $B_{s}=m v_{s} / e \lambda$. For each pair of fixed $a_{i}$ $(i=1,2)$ and $a_{s}$ the transversal relative velocity is determined by $v_{0 i \perp} / v_{s}=a_{i} / a_{s}$ (or $v_{e \perp} / v_{s}=a / a_{s}$ for ion-electron collisions). The CTMC results are indicated by the curves with filled or open symbols and the corresponding second 

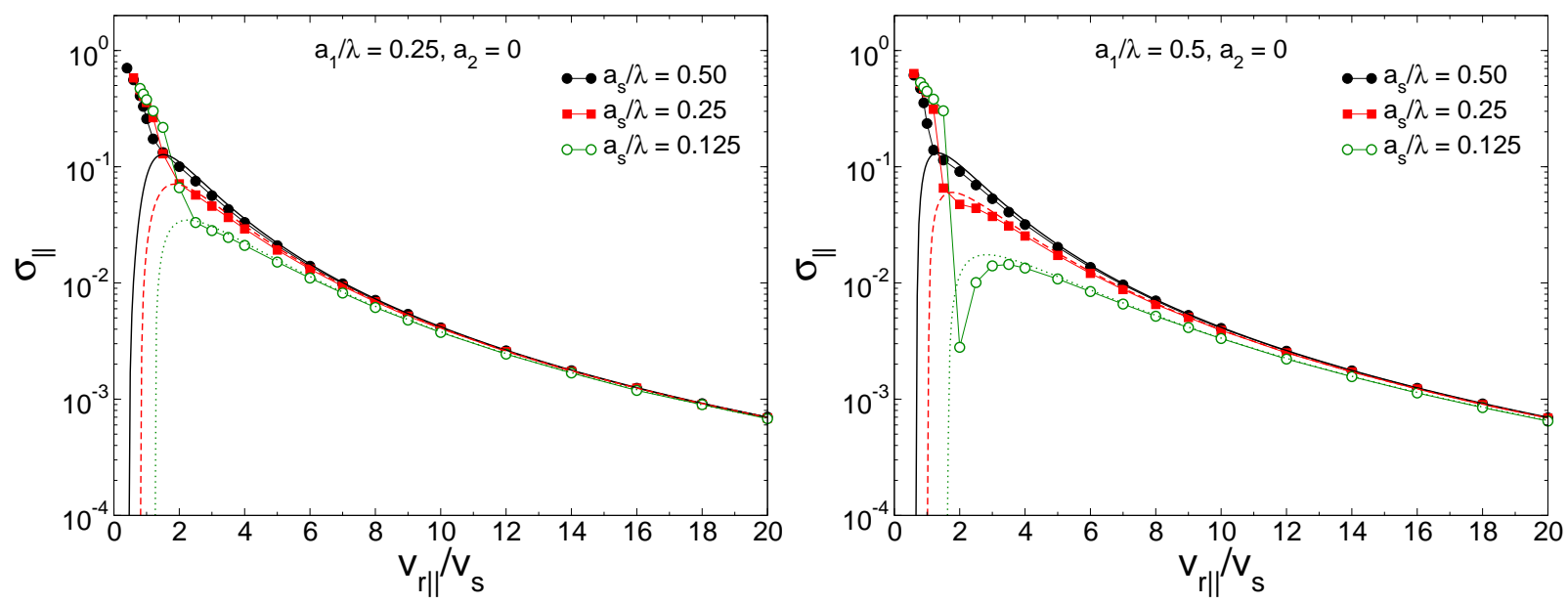

FIG. 8: (Color online) Same as in Fig. 7 but for $a_{1} / \lambda=0.25$ (left panel) and $a_{1} / \lambda=0.5$ (right panel). In left and right panels, $a_{s} / \lambda=0.5$ (solid), $a_{s} / \lambda=0.25$ (dashed), $a_{s} / \lambda=0.125$ (dotted).
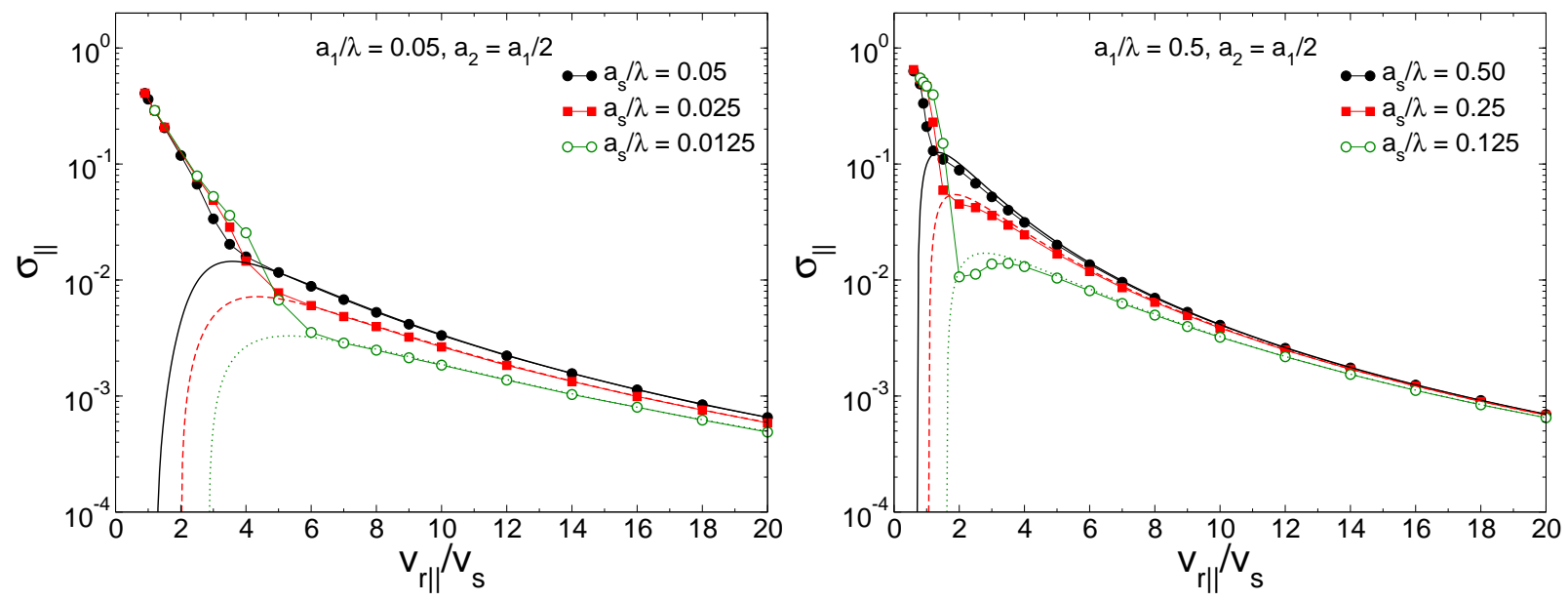

FIG. 9: (Color online) Same as in Fig. 7 but for $a_{2}=a_{1} / 2$ with $a_{1} / \lambda=0.05$ (left panel) and $a_{1} / \lambda=0.5$ (right panel). Left panel, $a_{s} / \lambda=0.05$ (solid curve), $a_{s} / \lambda=0.025$ (dashed curve), $a_{s} / \lambda=0.0125$ (dotted curve). Right panel, $a_{s} / \lambda=0.5$ (solid), $a_{s} / \lambda=0.25$ (dashed), $a_{s} / \lambda=0.125$ (dotted).

order predictions are given by the curves without symbols. Both the CTMC and second order calculations have been done for a regularized potential $U_{\mathrm{R}}$ with $\nu_{0}=5 \times 10^{-5}$. Note also the logarithmic scales for the cross-sections in Figs. 2, 3] and 6, 11]

First we discuss some general observations of the behavior of the cross-sections. In Figs. 2, 3 and 6 11 we can clearly observe that in the regimes of large relative velocities $v_{r \|} / v_{s} \gtrsim \kappa$ (where typically $2 \leqslant \kappa \leqslant 4$ ) the second order perturbative treatment agrees perfectly with the numerical CTMC results. In addition in the limit of very large velocities $v_{r \|} / v_{s} \gg 1$ the cross-sections $\sigma_{i}$ and $\sigma_{\|}$calculated either within perturbation theory or CTMC method with different strength of the magnetic field and transversal velocities converge to the same value. This behavior agrees with the predictions of the asymptotic expression (64) which is independent on $B$ and $v_{01 \perp}, v_{02 \perp}$ (or $v_{e \perp}$ ). It can also be seen that the smaller the transversal velocities, the better is the convergence to the regime of Eq. (64) . At large relative velocities the second order and CTMC cross-sections $\sigma_{\perp}$ shown in Figs. 10 and 11 agree with Eq. (80) with the asymptotic expression (65). Since at $v_{r \|} / v_{s} \gg 1$ the quantity $\sigma_{\perp}$ is not affected by the magnetic field but behaves as $\sigma_{\perp} \sim v_{01 \perp}^{2}, v_{02 \perp}^{2}$ for fixed electron cyclotron radii it will be larger for smaller $a_{s}$ (more precisely $\sigma_{\perp} \sim a_{s}^{-2}$ ) as shown in Figs. 10 and 11 .

At small velocities with $v_{r \|} / v_{s} \lesssim 1$ the second order treatment considerably deviates from CTMC simulations, see Figs. 1 13 and Figs. 6011 Here the second order cross-sections are given by approximate expressions (B6) and (B12) where the parameter $\varkappa$ at small relative velocities is given by $\varkappa \simeq \varkappa(0)=1+\lambda / \lambda(0)$ and $\lambda(0)$ is the dynamical cutoff $\lambda\left(v_{r \|}\right)$ Eq. (82) at $v_{r \|}=0$. Note that at finite cyclotron radii of the particles the quantity $\lambda(0)$ is a constant 

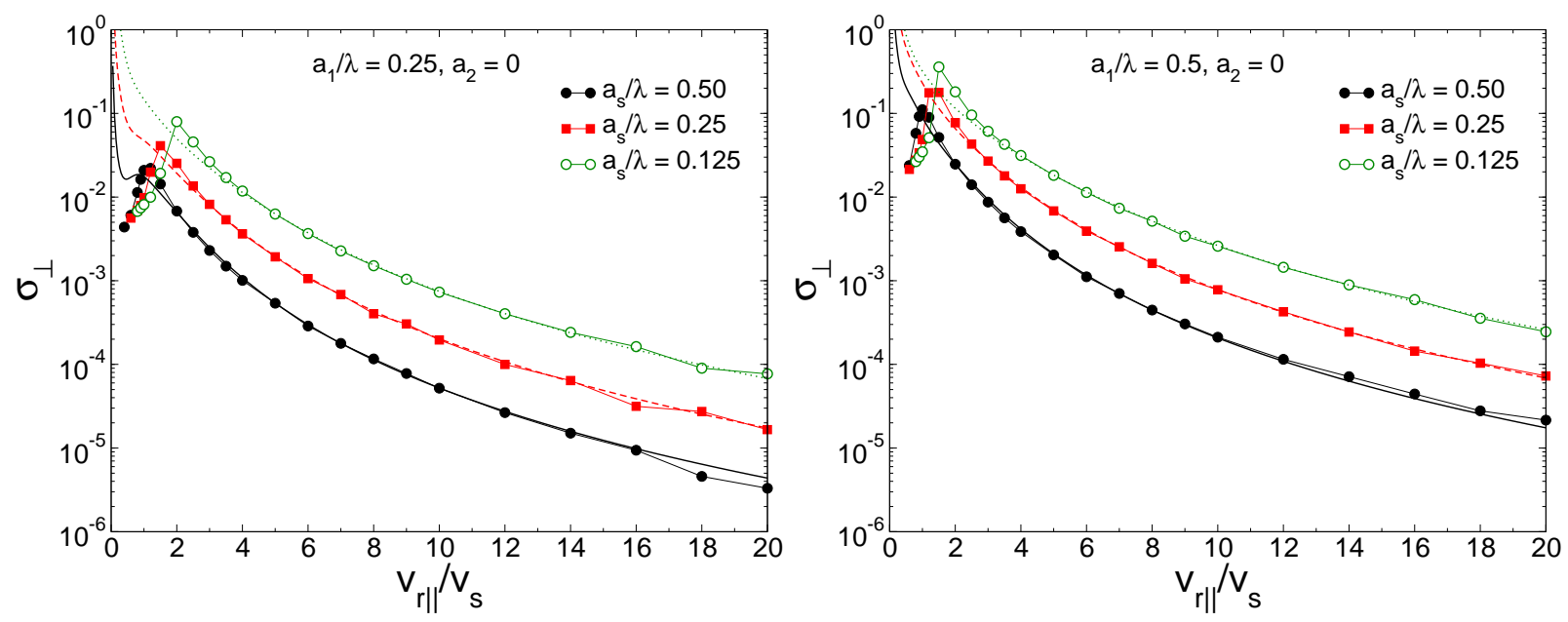

FIG. 10: (Color online) The cross-section $\sigma_{\perp}$ Eq. (80) for electron-electron collision in terms of dimensionless relative velocity component $v_{r \|} / v_{s}$ for $a_{2}=0$ and at $a_{1} / \lambda=0.25$ (left panel) and $a_{1} / \lambda=0.5$ (right panel). The curves with and without symbols correspond to CTMC simulations and the second order perturbative treatment, respectively. In left and right panels, $a_{s} / \lambda=0.5$ (solid curve), $a_{s} / \lambda=0.25$ (dashed), $a_{s} / \lambda=0.125$ (dotted).
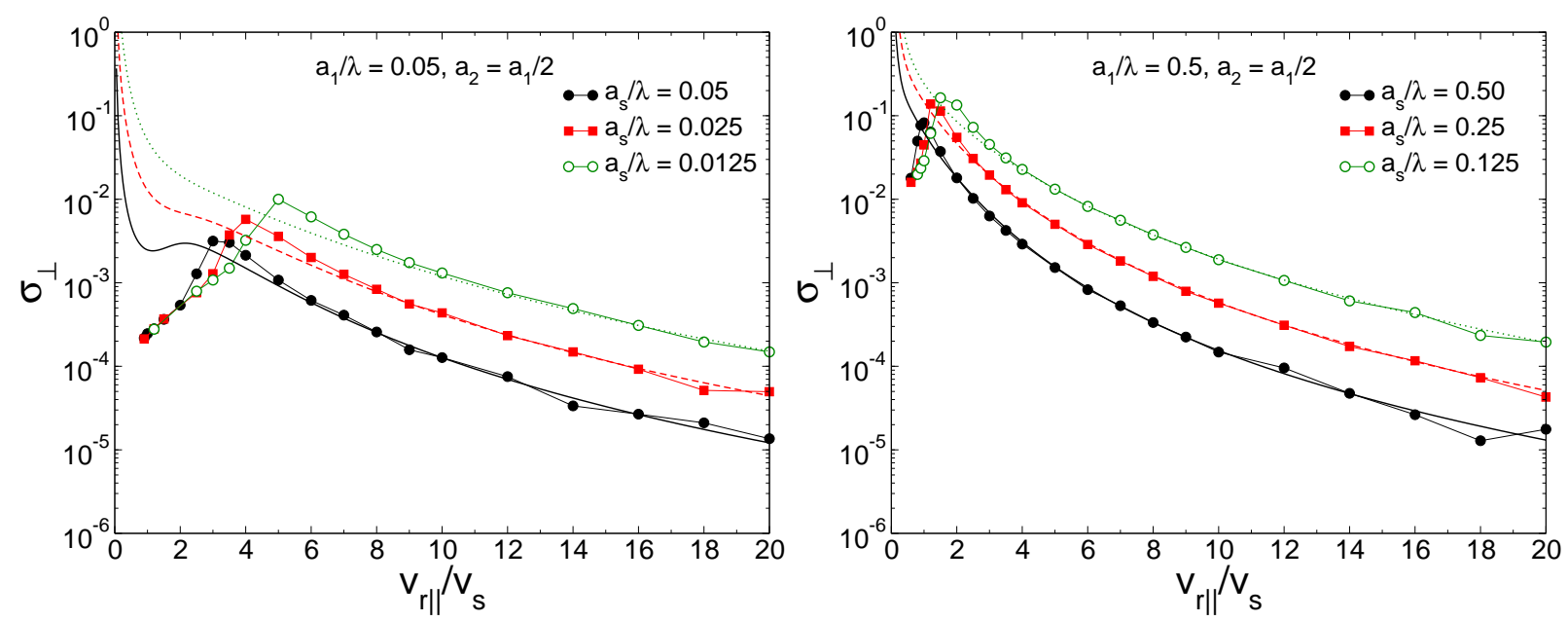

FIG. 11: (Color online) Same as in Fig. 10 but for $a_{2}=a_{1} / 2$ and at $a_{1} / \lambda=0.05$ (left panel) and $a_{1} / \lambda=0.5$ (right panel). Left panel, $a_{s} / \lambda=0.05$ (solid curve), $a_{s} / \lambda=0.025$ (dashed), $a_{s} / \lambda=0.0125$ (dotted). Right panel, $a_{s} / \lambda=0.5$ (solid), $a_{s} / \lambda=0.25$ (dashed), $a_{s} / \lambda=0.125$ (dotted).

depending on the value of $\nu_{0}$ and the transversal velocities. However, for vanishing cyclotron radii (as, e.g. in the left panels of Figs. 1, 2, 6] and 7) the cutoff parameter (82) at small velocities behaves as $\lambda / \lambda \sim\left(v_{s} / v_{r \|}\right)^{2}$. The quantity $(\varkappa-1)^{2}$ involved in Eqs. (B6) and (B12) falls as $\sim\left(v_{r \|} / v_{s}\right)^{4}$. This results in a strong self-cutting at small velocities. Thus employing the cutoff (82) the second order cross-section $\sigma_{\|}$is strongly reduced and decreases as $\sigma_{\|} \sim v_{r \|}^{5}$ and $\sigma_{\|} \sim v_{r \|}^{6}$ at $a=0$ and $a \neq 0$, respectively. The transversal cross-section $\sigma_{\perp}$, i.e. the second term in Eq. (B6), does not contain a term $(\varkappa-1)^{2}$ and diverges as $\sigma_{\perp} \sim v_{r \|}^{-2}$, see Figs. 10 and 11 In this small velocity regime the second order perturbative treatment is clearly invalid and a non-perturbative description is required.

In Figs. 4 and 5 we demonstrate the cross-section $\sigma_{i}$ for repulsive $(Z<0)$ ion-electron interaction. The dashed curves represent the cross-sections obtained from simple model considered in Sec. $\mathrm{V}$, see Eq. (69), with constant cutoff parameter $\nu_{0}$. Let us recall that the model (69) completely ignores the cyclotron motion of the particles. It is seen that in Figs. 4 and 5 (left panel) the agreement with CTMC simulations is quite satisfactory even for finite cyclotron radius $a$ and magnetic field. However, with increasing $a$ the CTMC simulations show a more involved picture as e.g. in Fig. 5 (right panel) than the predictions of a simple model (69). And the deviations from CTMC becomes more pronounced with increasing electron transversal velocity $v_{e \perp}$ and magnetic field, see e.g. open symbols in the right 
panel of Fig. 5. In the CTMC simulations the cross-section shrinks strongly at $v_{r \|} \gtrsim v_{s}$ with increasing relative velocity. This feature can be explain on the basis of a simple model discussed in Sec. $\nabla$. At strong but finite magnetic field the hard collisions like backscattering events may also occur but the total relative energy $\mu v_{r \|}^{2} / 2$ in Eq. (66) will be replaced here by the total energy $\mu\left(v_{r \|}^{2}+v_{e \perp}^{2}\right) / 2$. This will reduce the domain of the backscattering events (regime II introduced in Sec. V). With increasing $v_{r \|}$ this domain will be further shrunk and finally the scattering may occur only in the regime I where a strong magnetic field may strongly reduce the energy transfer. (Let us recall that the ion moves along the magnetic field. In this case the energy transfer vanishes with increasing $B$ for symmetry reason, see Refs. 14, 20]). Obviously this effect is pronounced by increasing transversal velocity as shown in Fig. [5 The similar feature is observed also for electron-electron interactions shown in the right panels of Figs. 8 and 9 .

For ion-electron collisions we also compare the cross-sections for attractive $(Z>0)$ and repulsive $(Z<0)$ interactions in Fig. 6. The solid curves represent the second order energy transfer which is quadratic in $Z$. For small velocities the cross-section $\sigma_{i}$ for repulsive interaction considerably exceeds the cross-section for attractive interaction. This is because of the backscattering events at $Z<0$ with large energy-velocity transfers. However, with increasing relative velocity the agreement between second order theory and CTMC and between two CTMC with positive and negative $Z$ continuously improves and is almost perfect over the full range of the velocity at $v_{r \|} / v_{s} \gtrsim 1$.

As has been emphasized in the preceding sections there are similarities between electron-electron and ion-electron (with $\mathbf{v}_{i}=v_{i \|} \mathbf{b}$ ) interactions but the energy transfers are not quite the same. In particular, the approximate relation $\sigma_{i} \simeq 2 \sigma_{\|}$for the negative ions with $Z<0$ may be violated at small velocities where the electron-electron crosssection $\bar{\sigma}_{\|}(\varphi)$ is not in general isotropic with respect to $\varphi$. In Figs. 7 and 8 the cyclotron radius of the second electron vanishes $a_{2}=0$ and thus $a=a_{1}$ as well as $\bar{\sigma}_{\|}(\varphi)$ are isotropic. In this case the relation between the cross-sections is exact $\sigma_{i}=2 \sigma_{\|}$. In Fig. 9 we show an anisotropic case where $a_{2}=a_{1} / 2$ and $a_{1}$ varies from a small (left panel) to a large value (right panel). Another feature which is absent in ion-electron collisions is the $\mathrm{cm}$ cyclotron motion for two-electron collisions which causes the transversal energy transfer $\sigma_{\perp}$ shown in Figs. 10] and 11. For symmetry reason this quantity vanishes at $a_{1}=a_{2}$ both in CTMC simulations (within the unavoidable numerical fluctuations) and the second order theory, see Eq. (52). In the examples shown in Figs. 10] and 11 the cyclotron radius $a_{2}$ of the second electron is smaller $a_{1}, a_{2}<a_{1}$. Since the cross-section $\sigma_{\perp}$ is positive the transverse energy of the first electron with $a_{1}>a_{2}$ transfers to the energy of parallel and transverse motion of the second electron.

\section{CONCLUSION}

In this paper we have investigated the binary collisions (BC) of two charged particles in the presence of constant magnetic field employing second order perturbation theory and classical trajectory Monte-Carlo (CTMC) simulations. Two distinct cases with symmetric and strongly asymmetric charges and masses of the particles have been considered in detail: (i) $\mathrm{BC}$ between two identical particles (e.g. electrons) and (ii) between an electron and heavy ion which moves with rectilinear trajectory (no cyclotron motion) along the magnetic field. Physically these two distinct cases are similar except the time-dependent center of mass cyclotron motion in the case of two identical particles. The second-order energy transfers for two-particles collision is calculated with the help of an improved BC treatment which is valid for any strength of the magnetic field and thus involves all cyclotron harmonics of the particles motion. For further applications (e.g., in cooling of ion beams, transport phenomena in magnetized plasmas) the actual calculations of the energy transfers have been done with a screened interaction potential which is regularized at the origin. The use of that potential can be viewed as an alternative to the standard cutoff procedure. For a repulsive ion-electron collisions we furthermore presented an exact solution for the energy transfer in the presence of an infinitely strong magnetic field. Here backscattering events may occur when the relative velocity transfer is independent on the strength of the Coulomb (regularized and screened) interaction $\Delta v_{\|}=-2 v_{r \|}$, where $v_{r \|}$ is the initial relative velocity of the guiding centers of the particles. The second order perturbative treatment is clearly invalid in this case. It has been shown that for repulsive interaction of the particles in a strong but finite magnetic field two scattering regimes must be distinguished with and without backscattering events with large energy-velocity transfers. This also suggests an improved perturbative treatment for repulsive interaction and in the case of strong magnetic field.

For checking the validity of the perturbative approach and also for applications beyond the perturbative regime we have employed numerical CTMC simulations. These CTMC calculations have been performed in a wide range of parameters (magnetic field and the relative velocities of the particles) and for a small regularization parameter, that is, for an interaction which is rather close to Coulomb at short distances. Within the second order treatment we have introduced a dynamical cutoff parameter which substantially improves the agreement of the theory with CTMC simulations. From a comparison with the non-perturbative CTMC simulations we have found as a quite general rule which is widely independent of the magnetic field strength that the predictions of the second order perturbative treatment are very accurate for $v_{r \|} / v_{s} \gtrsim 4$ for all studied parameters and cases, with the characteristic velocity $v_{s}$ given by Eq. (78). In contrast, for low relative velocities $v_{r \|} / v_{s} \lesssim 1$ the results obtained from perturbation 
theory strongly deviate from the CTMC simulations. Moreover in this regime the CTMC calculations display a large difference between positive and negative ions which disappears for high velocities $v_{r \|}$. Such a difference is completely absent in the second order perturbative treatment where the energy transfers is quadratic in the ion charge $Z e$. We have also tested the exact analytical model Eq. (69) derived for repulsive interaction and an infinitely strong magnetic field by comparing it in Figs. 4 and 5 with the CTMC simulations and found that the agreement is rather satisfactory even for finite (but strong) magnetic fields.

We believe that our theoretical findings will be useful for the interpretation of experimental investigations. Here, it is of particular interest to study some macroscopic physical quantities on the basis of the presented theoretical model such as cooling forces in storage rings and traps, stopping power of ion beams as well as transport coefficients in strongly magnetized plasmas. These studies require an average of the energy or velocity transfers with respect to the velocity distribution of the electrons. The cooling forces obtained by the perturbative approach are expected to be quite accurate if the low velocity regime only slightly contributes to the $\mathbf{v}_{r}$-average over $\langle\Delta E\rangle$. That is, if the typical $v_{r \|}$, given by the maximum of the thermal electron velocity and the ion velocity, are large compared to $v_{s}$, as it is usually the case for e.g. electron cooling in storage rings.

Another interesting issue not considered here is the interaction of the magnetized electrons with light ions (in particular with positrons, protons and antiprotons) when the magnetic field is so strong that the cyclotron motion of the ion cannot be neglected anymore. In this case the relative and center of mass motions are coupled to each other and the center of mass velocity of the particles cannot be represented in the form of simple cyclotron motion, Eq. (6). A detailed comparison with cooling force measurements and a study of other aspects are in progress and the results will be reported elsewhere.

\section{Acknowledgments}

One of the authors, H.B.N., is grateful for the support of the Alexander von Humboldt Foundation, Germany, and National Academy of Sciences of Armenia. This work was supported by the Bundesministerium für Bildung und Forschung (BMBF) under contract 06ER145 and by the Gesellschaft für Schwerionenforschung (GSI, ER/TOE). Discussions with C. Toepffer are gratefully acknowledged.

\section{APPENDIX A: DERIVATION OF THE COULOMB LOGARITHM $\Lambda(u)$}

In this Appendix we evaluate the effective cross section in the case of vanishing relative cyclotron radius. Using the definition of $\bar{\sigma}(\varphi)$, Eq. (33), and the energy transfer Eq. (50) we obtain

$$
\bar{\sigma}(\varphi)=-\frac{4 q^{4} \phi^{4} V_{0 \|}}{m v_{r \|}^{3}} \Lambda(u) .
$$

Here $u=\chi_{1} / \kappa_{1}$ and we have introduced the generalized Coulomb logarithm

$$
\Lambda(u)=\int_{0}^{\infty}\left[K_{1}(s)-u K_{1}(u s)\right]^{2} s d s .
$$

The integration in Eq. A2 can be done using the indefinite integrals of the functions $s K_{1}^{2}(s), s K_{1}^{2}(u s)$ and $s K_{1}(s) K_{1}(u s)$ [24]. This yields

$$
\begin{aligned}
\Lambda(u)= & \left\{-\frac{s^{2}}{2} K_{1}^{2}(s)-\frac{u^{2} s^{2}}{2} K_{1}^{2}(u s)+K_{0}(u s)\left[\frac{u^{2} s^{2}}{2} K_{2}(u s)-\frac{2 u^{2} s K_{1}(s)}{u^{2}-1}\right]\right. \\
& \left.+K_{0}(s)\left[\frac{s^{2}}{2} K_{2}(s)+\frac{2 u s K_{1}(u s)}{u^{2}-1}\right]\right\}_{s \rightarrow 0} \\
= & \left\{K_{0}(u s)\left[\frac{u^{2} s^{2}}{2} K_{2}(u s)-1+\frac{2 u^{2}\left(1-s K_{1}(s)\right)}{u^{2}-1}\right]\right. \\
& \left.+K_{0}(s)\left[\frac{s^{2}}{2} K_{2}(s)-1-2 \frac{1-u s K_{1}(u s)}{u^{2}-1}\right]\right\}_{s \rightarrow 0}+\frac{u^{2}+1}{u^{2}-1}\left[K_{0}(s)-K_{0}(u s)\right]_{s \rightarrow 0}-1 .
\end{aligned}
$$

Since at $s \rightarrow 0$ the modified Bessel functions behave as (see, e.g., 24]) $K_{2}(s)=2 / s^{2}+\mathrm{O}(1), K_{1}(s)=1 / s+\mathrm{O}(s \ln s)$, $K_{0}(s)=\ln (2 / s)+\mathrm{O}(1)$, the expression in the large brackets in the last line of Eq. (A3 vanishes as $s^{2} \ln ^{2} s \rightarrow 0$. 
There remains

$$
\Lambda(u)=\frac{u^{2}+1}{u^{2}-1}\left[K_{0}(s)-K_{0}(u s)\right]_{s \rightarrow 0}-1 .
$$

Since $K_{0}(s)=\ln (2 / s)+\mathrm{O}(1)$ at $s \rightarrow 0$ from Eq. A4 we finally obtain

$$
\Lambda(u)=\frac{u^{2}+1}{u^{2}-1} \ln u-1 .
$$

\section{APPENDIX B: THE ENERGY TRANSFER IN A SMALL VELOCITY LIMIT}

For the second order BC treatment the most critical situation is the small velocity regime where we expect some deviations from the non-perturbative CTMC simulations. For the improvement of the theoretical approach it is therefore imperative to investigate the energy transfer in the small velocity limit, $\left|v_{r \|}\right| \ll v_{0 \perp}$ or alternatively $\delta \ll a$. In principle this limit can be evaluated using the integral representation of the cross-sections, Eqs. (51), (52), (57) and (58). However, while these expressions are very convenient to calculate the high velocity limit of the energy transfers (see Sec. IV) they are not adopted for the evaluation of the small velocity limit due to the oscillatory nature of the function $R(t)$ at $v_{r \|} \rightarrow 0$. In this Appendix we consider instead an alternative but equivalent expressions for the effective cross-sections. For the axially symmetric interaction potential the effective cross-sections can be evaluated using Eqs. (30), (38), (41) and (43). We refer the reader to Refs. [14, 20] for details. The integration of these expressions with respect to the impact parameter $s$ yields the two-dimensional $\delta$-function, $\delta(\mathbf{Q})=\delta\left(\mathbf{k}_{\perp}+\mathbf{k}_{\perp}^{\prime}\right)$. Combining this function with the one-dimensional $\delta$-function $\delta\left(k_{\|}+k_{\|}^{\prime}\right)$ in Eqs. (30), (38) and (41) yields a threedimensional $\delta$-function $\delta\left(\mathbf{k}+\mathbf{k}^{\prime}\right)$ and the $\mathbf{k}^{\prime}$-integration in the energy transfers can be performed exactly. Furthermore it can be shown that only the imaginary part $\operatorname{Im}\left[G_{n}(\mathbf{k},-\mathbf{k})\right]$ contributes to the cross-sections. From Eq. (31) follows that the imaginary part of $G_{n}(\mathbf{k},-\mathbf{k})$ is expressed by the Dirac functions $\delta\left(\zeta_{n}(\mathbf{k})\right)$ which allows to perform the $k_{\|}$-integration. The final result reads

$$
\begin{gathered}
\bar{\sigma}(\varphi)=-\frac{4 q^{4} \phi^{4}}{m v_{r \|}^{3}} \sum_{n=0}^{\infty} \eta_{n}\left\{\frac{2 n^{2}}{\delta^{2}} \bar{V}(\varphi)\left[3 \Phi_{n ; 1}\left(k_{\|}, a\right)+k_{\|} \frac{\partial}{\partial k_{\|}} \Phi_{n ; 1}\left(k_{\|}, a\right)+\frac{\delta^{2}}{a} \frac{\partial}{\partial a} \Phi_{n ; 1}\left(k_{\|}, a\right)\right]\right. \\
\left.+\frac{v_{r \|}}{2} f(\varphi)\left[\frac{2 n^{2}}{\delta^{2}} \Phi_{n ; 1}\left(k_{\|}, a\right)+\Phi_{n-1 ; 3}\left(k_{\|}, a\right)+\Phi_{n+1 ; 3}\left(k_{\|}, a\right)\right]\right\}_{k_{\|}=n / \delta}, \\
\bar{\sigma}_{r \perp}(\varphi)=\frac{8 q^{4} \phi^{4} \omega_{c}^{2}}{m v_{r \|}^{4}} \sum_{n=1}^{\infty} n^{2}\left\{2 \Phi_{n ; 1}\left(k_{\|}, a\right)+k_{\|} \frac{\partial}{\partial k_{\|}} \Phi_{n ; 1}\left(k_{\|}, a\right)+\frac{\delta^{2}}{a} \frac{\partial}{\partial a} \Phi_{n ; 1}\left(k_{\|}, a\right)\right\}_{k_{\|}=n / \delta} \\
\bar{\sigma}_{r 1}(\varphi)=\frac{8 q^{4} \phi^{4}}{\delta^{2}} \sum_{n=1}^{\infty}\left[n^{2} \Phi_{n ; 1}\left(k_{\|}, a\right)\right]_{k_{\|}=n / \delta},
\end{gathered}
$$

where the function $\Phi_{n ; m}\left(k_{\|}, a\right)$ is defined as

$$
\Phi_{n ; m}\left(k_{\|}, a\right)=\frac{(2 \pi)^{4}}{4} \int_{0}^{\infty} U^{2}\left(k_{\|}, k_{\perp}\right) J_{n}^{2}\left(k_{\perp} a\right) k_{\perp}^{m} d k_{\perp} .
$$

In Eq. (B1) we have introduced the notations $\eta_{n}=1-\frac{1}{2} \delta_{n 0}, \bar{V}(\varphi)=V_{0 \|}-\left(v_{r \|} / 2\right) f(\varphi)$ with $f(\varphi)=\left(a_{1}^{2}-a_{2}^{2}\right) / a^{2}(\varphi)$.

Equations (B1)-(B3) are equivalent to the integral representations (34)-(36), (47) and (48) of the cross-sections, respectively. The function $\Phi_{n ; m}\left(k_{\|}, a\right)$ is taken at $k_{\|}=n / \delta$ which in the limit of small velocities becomes very large (except the term with $n=0$ in Eq. (B1)). For the regularized interaction potential (49) from Eq. (B4) at $k_{\|} a 1$ we obtain

$$
\Phi_{n ; 1}\left(k_{\|}, a\right) \simeq \frac{5 \lambda^{3}}{32 a} \frac{\left(\varkappa^{2}-1\right)^{2}}{\left(k_{\|} \lambda\right)^{7}}, \quad \Phi_{n ; 3}\left(k_{\|}, a\right) \simeq \frac{\lambda}{32 a} \frac{\left(\varkappa^{2}-1\right)^{2}}{\left(k_{\|} \lambda\right)^{5}} .
$$


Substituting these expressions into Eqs. (B1)-(B2) in the lowest order with respect to $v_{r \|}($ or $\delta$ ) we arrive at

$$
\begin{gathered}
\bar{\sigma}(\varphi) \simeq \frac{q^{4} \phi^{4}}{m}\left[\frac{\left|v_{r \|}\right|}{v_{r \|}} \frac{5 \zeta(5) V_{0 \|}}{\left(\omega_{c} \lambda\right)^{3}}\left(\varkappa^{2}-1\right)^{2}\left(\frac{\delta}{\lambda}\right)^{2} \frac{\lambda}{a}-\frac{2}{v_{r \|}^{2}} f(\varphi) \psi\left(\frac{a}{\lambda}\right)\right], \\
\bar{\sigma}_{r \perp}(\varphi) \simeq-\frac{25 \zeta(5) q^{4} \phi^{4}}{4 m \omega_{c}^{2} \lambda^{2}}\left(\varkappa^{2}-1\right)^{2}\left(\frac{\delta}{\lambda}\right)^{3} \frac{\lambda}{a}, \\
\bar{\sigma}_{r 1}(\varphi) \simeq \frac{5 \zeta(5) q^{4} \phi^{4}}{4}\left(\varkappa^{2}-1\right)^{2}\left(\frac{\delta}{\lambda}\right)^{5} \frac{\lambda}{a} .
\end{gathered}
$$

Here $\zeta(z)$ is the Riemann function with $\zeta(5) \simeq 1.0369$. The function $\psi(u)$ is expressed by the modified Bessel functions

$$
\begin{aligned}
\psi(u)= & \frac{1}{2 u} \frac{\partial}{\partial u}\left\{u^{2}\left[I_{1}(u) K_{1}(u)+I_{1}(\varkappa u) K_{1}(\varkappa u)\right]\right\} \\
& +\frac{2}{\varkappa^{2}-1}\left[I_{1}(u) K_{1}(u)-\varkappa^{2} I_{1}(\varkappa u) K_{1}(\varkappa u)\right] .
\end{aligned}
$$

Note two limiting cases of this function. At $u \ll 1$ (vanishing cyclotron radius) and $\varkappa \rightarrow 1$ from Eq. (B9) we obtain

$$
\begin{gathered}
\psi(u) \simeq \frac{u^{2}}{8}\left(\varkappa^{2}+1-\frac{4 \varkappa^{2}}{\varkappa^{2}-1} \ln \varkappa\right), \\
\psi(u) \simeq\left(\varkappa^{2}-1\right)^{2} \frac{u^{3}}{48} \frac{\partial}{\partial u} \frac{1}{u} \frac{\partial}{\partial u} \frac{1}{u} \frac{\partial}{\partial u}\left[u^{2} I_{1}(u) K_{1}(u)\right],
\end{gathered}
$$

respectively. The case of ion-electron BC is easily recovered from the obtained expressions as described at the end of Sec. IV Note that the cm cyclotron motion is absent here and in Eqs. (B1) and (B6) the terms proportional to the function $f(\varphi)$ must be neglected.

From Eqs. (B6)-(B8) we then obtain that the relative transversal cross-sections at small relative velocities behave as $\bar{\sigma}_{r \perp}(\varphi) \sim v_{r \|}^{3}, \bar{\sigma}_{r 1}(\varphi) \sim v_{r \|}^{5}$. The first term in the cross-section $\bar{\sigma}(\varphi)$ vanishes as $\sim v_{r \|}^{2}$ while the second one which corresponds to the zero harmonic with $n=0$ in Eq. (B1) diverges as $\sim v_{r \|}^{-2}$. This later term vanishes at $a_{1}=a_{2}$ and predicts an infinitely large energy transfer at $a_{1}>a_{2}$ (or energy gain at $a_{1}<a_{2}$ ). As expected the cross-sections (B6) $-(\mathrm{B} 8)$ are strongly anisotropic with respect to the phase $\varphi$.

Finally, we consider the small velocity limit of the cross-sections $\bar{\sigma}(\varphi)$ and $\bar{\sigma}_{r \perp}(\varphi)$ at vanishing cyclotron radius, $a=0$ (see Eq. (하) $)$,

$$
\bar{\sigma}(\varphi)=-\frac{V_{0 \|}}{v_{r \|}} \bar{\sigma}_{r \perp}(\varphi) \simeq-\frac{q^{4} \phi^{4} V_{0 \|} v_{r \|}}{3 m \omega_{c}^{4} \lambda^{4}}\left(\varkappa^{2}-1\right)^{2}
$$

In this case the cross-sections behave as $\bar{\sigma}(\varphi) \sim v_{r \|}$ and $\bar{\sigma}_{r \perp}(\varphi) \sim v_{r \|}^{2}$ at small relative velocity $v_{r \|}$ (cf. with Eqs. (B6) and (B7)).

[1] Y. S. Derbenev and A. N. Skrinsky, Part. Accel. 8, 1 (1977).

[2] Y. S. Derbenev and A. N. Skrinsky, Part. Accel. 8, 235 (1978).

[3] A. H. Sørensen and E. Bonderup, Nucl. Instrum. Methods. Phys. Res. 215, 27 (1983).

[4] H. Poth, Phys. Rep. 196, 135 (1990).

[5] I. N. Meshkov, Phys. Part. Nucl. 25, 631 (1994).

[6] in Proceedings of the 16th International Symposium on Heavy Ion Inertial Fusion, edited by C. Deutsch, M.-M. Gombert, G. Maynard, J.-P. Didelez, and E. Sonnendrücker (Saint Malo, France, 2007), [Nucl. Instrum. Methods Phys. Res. A 577 (2007)].

[7] H. Hasegawa, M. Robnik, and G. Wunner, Prog. Theor. Phys. Suppl. 98, 198 (1989).

[8] J. G. Siambis, Phys. Rev. Lett. 37, 1750 (1976). 
[9] M. E. Glinsky, T. M. O’Neil, M. N. Rosenbluth, K. Tsuruta, and S. Ichimaru, Phys. Fluids B 4, 1156 (1992).

[10] G. Zwicknagel, in Non-Neutral Plasma Physics III, edited by J. J. Bollinger, R. L. Spencer, and R. C. Davidson, AIP Conf. Proc. No. 498 (AIP, Melville, NY, 1999), p. 469.

[11] G. Zwicknagel, Theory and simulation of the interaction of ions with plasmas, thesis, University of Erlangen, Erlangen (2000), (http://www.opus.ub.uni-erlangen.de/opus/volltexte/2008/913/)

[12] G. Zwicknagel and C. Toepffer, in Non-Neutral Plasma Physics IV, edited by F. Anderegg, L. Schweikhard, and C. F. Driscoll, AIP Conf. Proc. No. 606 (AIP, Melville, NY, 2002), p. 499.

[13] G. Zwicknagel, in Beam Cooling and Related Topics, edited by S. Nagaitsev and R. J. Pasquinelli, AIP Conf. Proc. No. 821 (AIP, Melville, NY, 2006), p. 513.

[14] H. B. Nersisyan, C. Toepffer, and G. Zwicknagel, Interactions Between Charged Particles in a Magnetic Field: A Theoretical Approach to Ion Stopping in Magnetized Plasmas, (Springer, Heidelberg, 2007).

[15] M. C. Gutzwiller, in Chaos in Classical and Quantum Mechanics, edited by F. John, L. Kadanoff, J. E. Marsden, L. Sirovich, and S. Wiggins (Springer-Verlag, New York, 1990), pp. 323-339.

[16] G. Schmidt, E. E. Kunhardt, and J. L. Godino, Phys. Rev. E 62, 7512 (2000).

[17] B. Hu, W. Horton, C. Chiu, and T. Petrosky, Phys. Plasmas 9, 1116 (2002). Pap-49.tex

[18] D. K. Geller and C. Weisheit, Phys. Plasmas 4, 4258 (1997).

[19] C. Toepffer, Phys. Rev. A 66, 022714 (2002).

[20] H. B. Nersisyan, G. Zwicknagel, and C. Toepffer, Phys. Rev. E 67, 026411 (2003).

[21] A. I. Akhiezer, I. A. Akhiezer, R. V. Polovin, A. G. Sitenko, and K. N. Stepanov, Plasma Electrodynamics, vol. 1 (Pergamon, Oxford, 1975), 1st ed.

[22] G. Kelbg, Ann. Physik 12, 219 (1963).

[23] C. Deutsch, Phys. Lett. A 60, 317 (1977).

[24] I. S. Gradshteyn and I. M. Ryzhik, Tables of Integrals, Series and Products (Academic, New York, 1980), 2nd ed.

[25] R. Abrines and I. C. Percival, Proc. Phys. Soc. London 88, 861 (1966).

[26] Q. Spreiter and M. Walter, J. Comput. Phys. 152, 102 (1999).

[27] G. Zwicknagel, Simulations for Ion Traps - Methods and Numerical Implementation, in Trapped Charged Particles and Fundamental Interactions, edited by K. Blaum, F. Herfurth, Lecture Notes in Physics, Volume 749, pp. 69-96 (SpringerVerlag, Berlin, Heidelberg, 2008).

[28] G. S. Fishman, Monte Carlo (Springer, New York, 1999), 3rd ed.

[29] K. Binder and D. W. Heermann, Monte Carlo Simulation in Statistical Physics, 3rd edn (Springer, Berlin 1997).

[30] H. Hahn, E. A. Mason, and F. J. Smith, Phys. Fluids 14, 278 (1971). 